\title{
Islamic Republic of Afghanistan: Fifth Review Under the Three-Year Arrangement Under the Poverty Reduction and Growth Facility and Request for Waiver of Performance Criteria, and Rephasing and Extension of the Arrangement-Staff Report; Staff Statement; Press Release on the Executive Board Discussion; and Statement by the Executive Director for Islamic Republic of Afghanistan
}

In the context of the fifth review under the three-year arrangement under the Poverty Reduction and Growth Facility and a request for a waiver of performance criteria, and rephasing and extension of the arrangement, the following documents have been released and are included in this package:

- $\quad$ The staff report for the Fifth Review Under the Three-Year Arrangement Under the Poverty Reduction and Growth Facility and Request for Waiver of Performance Criteria, and Rephasing and Extension of the Arrangement, prepared by a staff team of the IMF, following discussions that ended on February 3, 2009, with the officials of Islamic Republic of Afghanistan on economic developments and policies. Based on information available at the time of these discussions, the staff report was completed on April 7, 2009. The views expressed in the staff report are those of the staff team and do not necessarily reflect the views of the Executive Board of the IMF.

- $\quad$ A staff statement of April 22, 2009, updating information on recent developments.

- $\quad$ A Press Release summarizing the views of the Executive Board as expressed during its Board meeting April 22, 2009, discussion of the staff report that completed the review.

- A statement by the Executive Director for the Islamic Republic of Afghanistan.

The documents listed below has been separately released.

Letter of Intent sent to the IMF by the authorities of the Islamic Republic of Afghanistan* Memorandum of Economic and Financial Policies by the authorities of the Islamic Republic of Afghanistan*

Technical Memorandum of Understanding*

*Also included in Staff Report

The policy of publication of staff reports and other documents allows for the deletion of market-sensitive information.

Copies of this report are available to the public from

International Monetary Fund • Publication Services

$70019^{\text {th }}$ Street, N.W. • Washington, D.C. 20431

Telephone: (202) 623-7430 • Telefax: (202) 623-7201

E-mail: publications@imf.org • Internet: http://www.imf.org

\section{International Monetary Fund Washington, D.C.}





\section{INTERNATIONAL MONETARY FUND}

\section{ISLAMIC REPUBLIC OF AFGHANISTAN}

\section{Fifth Review Under the Three-Year Arrangement Under the Poverty Reduction and Growth Facility and Request for Waiver of Performance Criteria, and Rephasing and Extension of the Arrangement}

\section{Prepared by the Middle East and Central Asia Department}

(In consultation with other Departments)

\section{Approved by Adam Bennett and Dominique Desruelle}

April 7, 2009

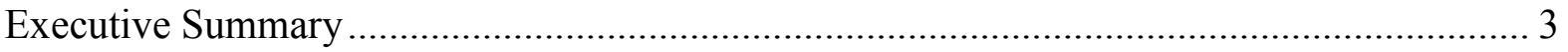

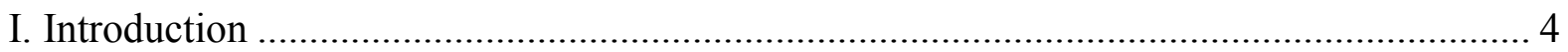

II. Recent Developments and Performance under the Program............................................... 5

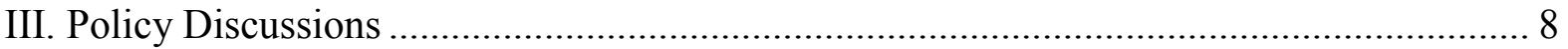

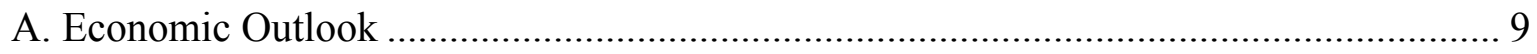

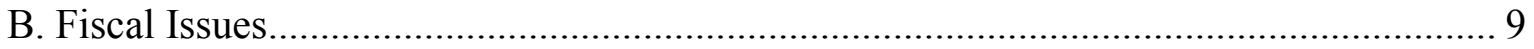

C. Monetary and Financial Sector Issues...................................................................... 10

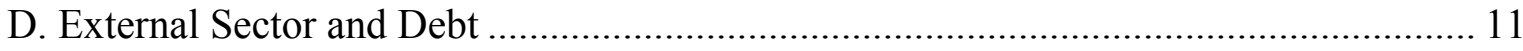

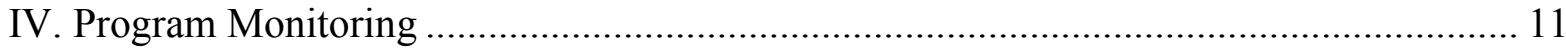

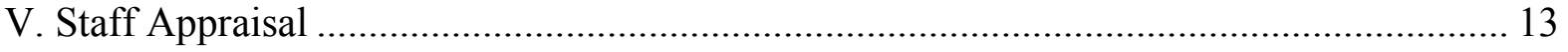

\section{Text Box}

1. Status of HIPC Completion Point Triggers ……….......................................................12

Text Table

Fiscal Performance. 


\section{Text Figures}

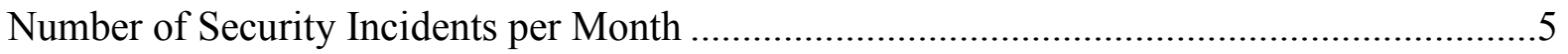

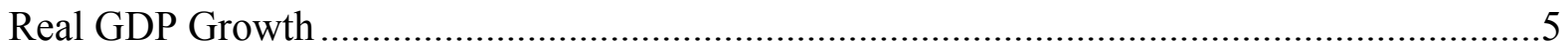

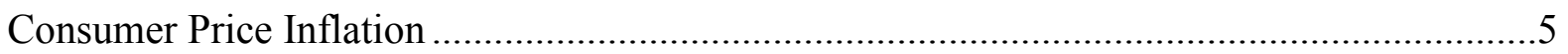

Monetary Aggregates ...............................................................................................

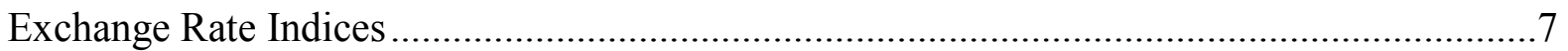

Figures

1. Output, Inflation, and External Sector Developments ..................................................15

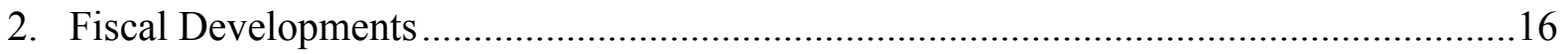

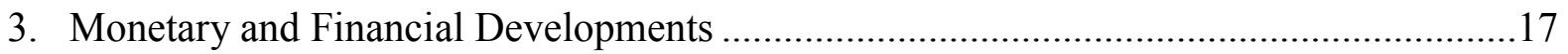

Tables

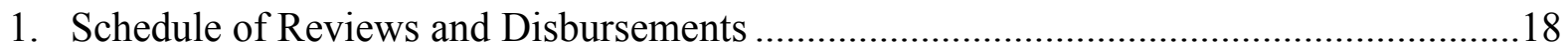

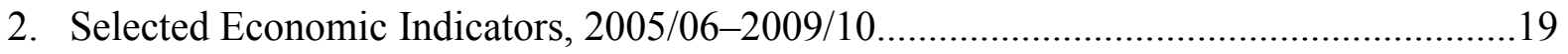

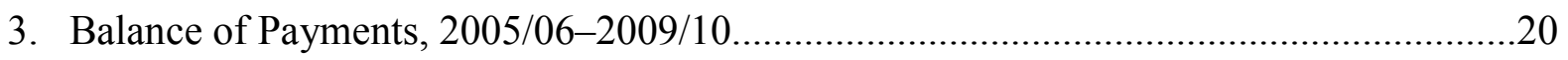

4a. Core Budget, 2005/06-2009/10 (in billions of Afghanis) ...............................................21

4b. Core Budget, 2005/06-2009/10 (in percent of GDP) .....................................................22

5 Monetary Program (Central Bank), 2007/08-2009/10 ...................................................23

6. Quantitative Performance Criteria and Indicative Targets under the PRGF

Arrangement, 2008/09 ....................................................................................24

7. Structural Conditionality under the PRGF Arrangement, June-December 2008 ..............25

8. Medium-Term Macroeconomic Framework, 2008/09-2013/14 ………………...............26

9. External Financing Requirements and Sources, 2006/07-2013/14 ..................................27

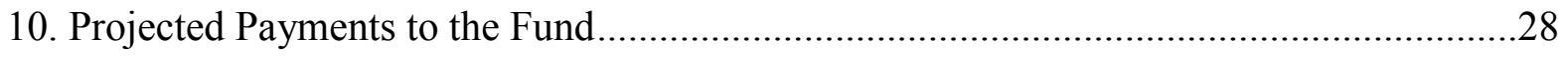

Attachments

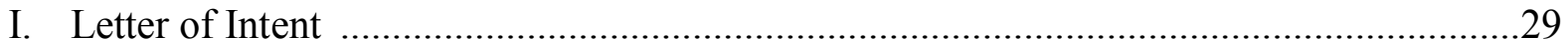

II. Supplementary Memorandum of Economic and Financial Policies for 2009/10 ...............31

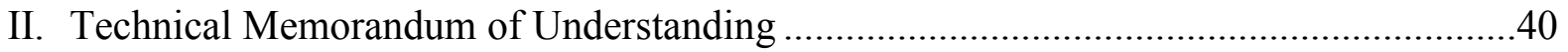




\section{EXECUTIVE SUMMARY}

During the past year, Afghanistan's economic, security, and political situation has become increasingly challenging. The economy experienced a drought and higher commodity prices in 2008, while security worsened, governance issues hampered revenue collection, and the political environment has turned uncertain ahead of Presidential elections in August.

Performance under the PRGF-supported program has been mixed. Monetary and financial policies were broadly consistent with the program. However, difficulties were evident in reforming public enterprises and in the fiscal area, where revenues were weaker than projected and expenditure overruns led to historically low cash balances.

The economic program for 2009/10 envisages a prudent monetary policy to ensure a fast disinflation, fiscal adjustment anchored on remedial measures to increase revenues and contain spending, and closer monitoring of public enterprises that pose fiscal risks.

The main policies and actions under the program are:

$>\quad$ A reduction in reserve money growth from 35 percent in 2008/09 to 17 percent in 2009/10 to lower inflation to about 6 percent by March 2010.

$>\quad$ Reforms to raise the revenue-to-GDP ratio from 6.7 percent in 2008/09 to 7.3 percent in 2009/10. The reforms include collecting the business receipts tax on imports, monitoring fuel imports through the state fuel company and private depots, and expanding post-clearance audits to additional customs posts.

$>\quad$ A contained spending envelope and prioritized public spending in the areas of security, health, and education to lower the operating budget deficit excluding grants from 5.5 percent of GDP in 2008/09 to 4.7 percent in 2009/10.

$>\quad$ Revamped efforts to reform public enterprises by finalizing the audits of the state fuel company, strengthening the monitoring of the state electricity company, and preparing an action plan for these and two other key public enterprises.

Based on the implementation of prior actions, a turnaround in revenue performance since January 2009, and the strength of the authorities' program for 2009/10, the staff recommends the completion of the Fifth Review. The staff also supports an extension of the arrangement until March 2010 to allow for the implementation of pending reforms. 


\section{INTRODUCTION}

1. Afghanistan faces enormous economic challenges, most notably to rebuild and reform its institutions, put its financing onto a sustainable path, and lay the foundation for growth and poverty reduction. This task has become increasingly difficult because of the growing insurgency and corruption. Social and economic conditions have improved in recent years, but poverty remains widespread, public enterprises pose fiscal risks, weak institutions and enforcement deter investment and deprive the government of resources, and domestic revenues cover one-third of public spending.

2. Afghanistan's economic program, supported by the Poverty Reduction and Growth Facility (PRGF), seeks to address these challenges by strengthening key economic institutions and governance and reforming public enterprises while ensuring economic stability and low inflation. ${ }^{1}$ Afghanistan also participates in the Heavily Indebted Poor Countries (HIPC) Initiative. Satisfactory implementation of the program and other conditions for debt relief will allow for the cancellation of nearly 100 percent of Afghanistan's foreign debt.

3. The IMF Executive Board concluded the Fourth Review under the PRGF arrangement on July 7, 2008. At that time, Executive Directors encouraged the authorities to focus monetary policy on containing the inflationary impact of higher fuel and food prices and to proceed with reforms of key public enterprises. Directors also stressed the importance of implementing revenue-enhancing measures to prevent continued aid dependence and achieve fiscal sustainability over the long term.

4. Discussions on the Fifth Review were held in Kabul during December 2-16, 2008 and January 25-February 3, 2009. ${ }^{2}$ In the attached Letter of Intent and Supplementary Memorandum of Economic and Financial Policies (Attachments I and II), the authorities request the completion of the Fifth Review and describe the policy framework for the 2009/10 fiscal year (March 21, 2009-March 20, 2010). The authorities also request an extension of the arrangement to March 2010 and a rephasing of disbursements to allow them more time to implement pending reforms and reach the completion point under the HIPC Initiative.

\footnotetext{
${ }^{1}$ The Executive Board of the IMF approved Afghanistan's three-year arrangement under the PRGF amounting to SDR 81.0 million (50 percent of quota) on June 26, 2006. The Fund has already disbursed SDR 58.4 million and SDR 11.3 million will be made available upon the completion of this review (Table 1).

${ }^{2}$ The staff met with Minister of Finance Ahady and his successor, Minister Zakhilwal; Governor Fitrat of the central bank; Minister of Commerce and Industry Shahrani; other government officials; and representatives from the private sector and the donor community. Messrs. Bennett and Gelbard (Middle East and Central Asia Department) headed the December and January missions, respectively. The staff teams comprised Mr. Ma, Ms. Mineshima, and Mr. Wieczorek (Middle East and Central Asia Department); Ms. Asmundson (Strategy, Policy, and Review Department); and Messrs. Gracia and Haider (Fiscal Affairs Department). Mr. Abdallah (the Fund's resident representative) assisted the missions.
} 


\section{Recent Developments and Performance Under the Program}

5. Security has been deteriorating and the political environment surrounding the program has become increasingly complex. The number of security incidents rose dramatically in 2008 and the government has been losing control in many provinces. Opposition from vested interests and governance problems have hampered policy implementation, especially in reforming customs administration and public enterprises. Presidential elections are scheduled for August 20, 2009.

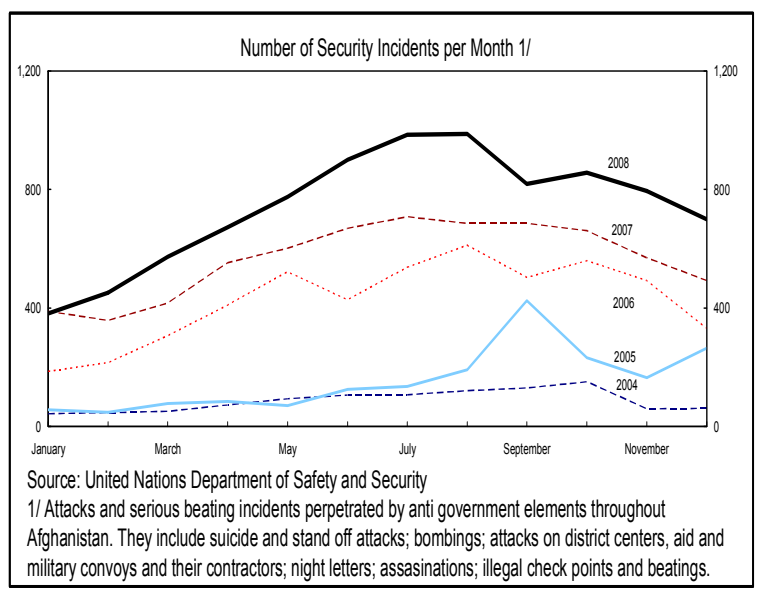

\section{In 2008, a drought and higher commodity prices reduced growth and drove up} inflation. Staff estimates that economic growth fell to about 3 percent in 2008/09 compared to 12 percent in 2007/08, mainly reflecting a sharp decline in agricultural output (Table 2). ${ }^{3}$ Outside the formal sector, the United Nations estimates that opium production fell because of low opium prices and efforts to discourage poppy production. ${ }^{4}$ The drought (which affected mainly cereals) and restrictions on wheat exports by regional trading partners added to domestic food price pressures and combined with higher fuel prices to push the 12-month rate of inflation to a peak of 43 percent in May 2008. Lower world prices since then allowed inflation to fall to 9 percent in February 2009. The external current account balance worsened because of emergency wheat imports (Table 3 ).
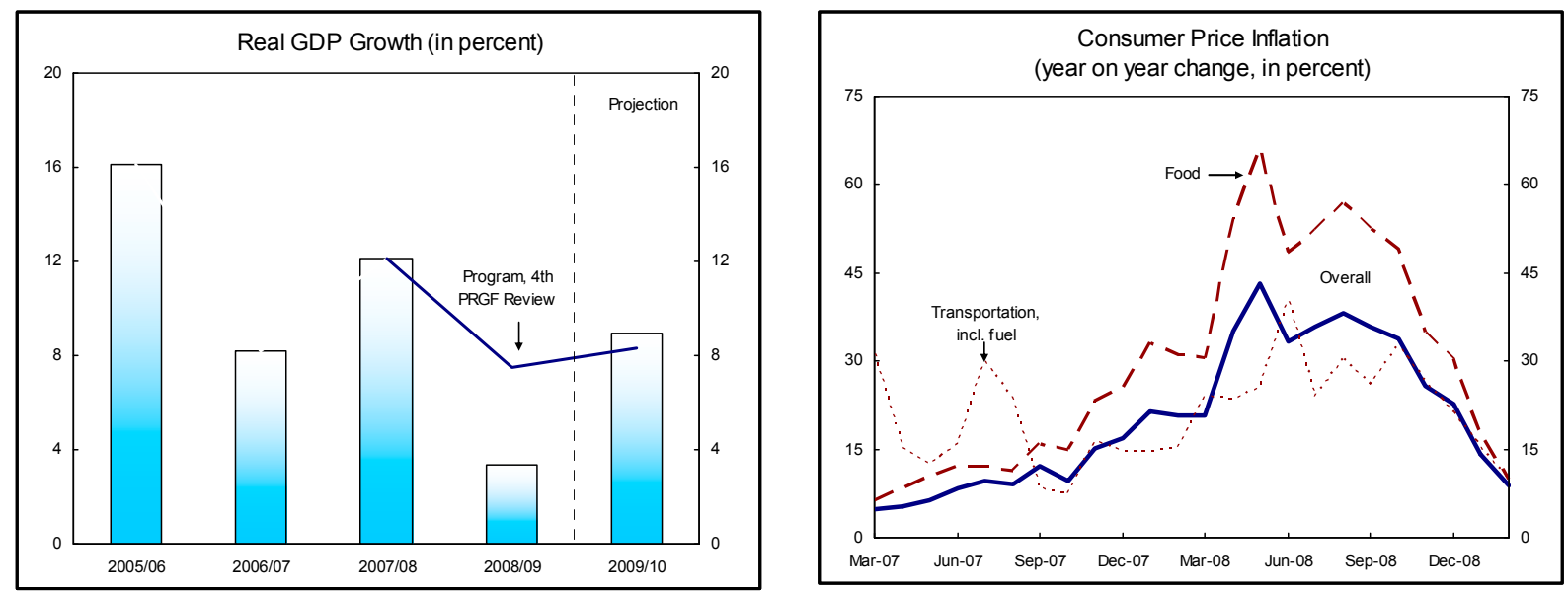

\footnotetext{
${ }^{3}$ Agriculture accounts for about 30 percent of the formal economy.

${ }^{4}$ GDP estimates exclude opium production.
} 
7. The fiscal outturn was worse than projected due to a weak revenue performance and higher spending. The revenue-to-GDP ratio fell and operating expenditures were higher than projected (Table 4$).{ }^{5}$ Preliminary estimates show that, despite higher than expected grants to the operating budget, the operating balance including grants in 2008/09 recorded a deficit of 1.1 percent of GDP, compared to a 0.5 percent surplus under the program. The overall budget balance also appears to have worsened by as much as 2.3 percent of GDP, and discretionary cash balances to have fallen from 0.8 percent of GDP $2007 / 08$ to 0.2 percent in $2008 / 09 .^{6}$

\section{8. $\quad$ Revenue performance faltered} because of governance problems at customs. Taxes on goods and services posted strong growth, mainly because of the strengthening of large taxpayers' offices (end-September 2008 structural benchmark). However, collection in regional offices was flat, and customs collection

\begin{tabular}{|lccr|}
\hline \multicolumn{4}{c|}{ Fiscal Performance } \\
\hline \multicolumn{4}{c}{ (in percent of GDP) } \\
\cline { 2 - 4 } & $2007 / 08$ & 7.0 & 6.7 \\
\cline { 2 - 4 } Domestic revenues & 6.9 & 2.8 & 2.3 \\
Customs & 2.7 & 4.2 & 4.4 \\
Other & 4.3 & 9.5 & 10.1 \\
Grants & 11.1 & 3.7 & 4.4 \\
Operating & 4.8 & 5.8 & 5.7 \\
Development & 6.3 & 19.1 & 20.9 \\
Total expenditures & 19.9 & 10.2 & 12.2 \\
Operating & 10.5 & 8.8 & 8.7 \\
Development & 9.4 & & \\
Overall balance & & -12.1 & -14.3 \\
Excluding grants & -12.9 & -2.5 & -4.1 \\
Including grants & -1.8 & & \\
Operating balance & & -3.2 & -5.5 \\
Excluding grants & -3.6 & 0.5 & -1.1 \\
Including grants & 1.2 & \\
Domestic revenues & & & \\
In billion of Afghanis & 33.4 & 44.5 & 40.0 \\
Source: Afghan authorities and Fund staff estimates. & \\
\hline
\end{tabular}
during the first three quarters of 2008/09 was lower than in the same period in 2007/08 despite higher import volumes. Collection turned around during January-March 2009, but not enough to affect the year total. ${ }^{7}$ Worsening security affected collection but governance problems played the key role, especially in relation to fuel imports handled by the state fuel company.

\section{Additional public spending was needed to cover unexpected outlays on food,} education, and defense. The midyear budget review provided for emergency wheat imports, higher teachers' salaries, additional military spending, and the settlement of education wage arrears. Wheat imports are not expected to recur in 2009/10. The teachers' salary increases are part of the accelerated implementation of a "pay and grade" reform already incorporated in the medium-term framework.

\footnotetext{
${ }^{5}$ Due to the weak fiscal performance, staff now expects that fiscal sustainability, defined as a balanced operating budget excluding grants, will not be attained at least until 2014/15, one year later than estimated at the time of the Fourth Review.

${ }^{6}$ Discretionary cash balances represent freely available government deposits; i.e., excluding funds earmarked for development projects and counterpart PRGF balances.

${ }^{7}$ During the last three months of the 2008/09 fiscal year (December 2008-March 2009), average monthly collection is estimated at Af 3.9 billion compared to Af 3 billion during the first 9 months of the year.
} 


\section{Monetary policy was in line with the program and the Afghani remained}

broadly stable. The growth of currency in circulation was kept within program ceilings. Reserve money exceeded the targets due to large deposits in the central bank from a commercial bank that handles coalition forces' expenditures (Table 5). To strengthen control over monetary aggregates, the central bank continued to foster the market for capital notes (central bank bills) and developed an electronic registry (end-September 2008 structural benchmark) to pave the way for a secondary market. ${ }^{8}$ The Afghani has remained broadly stable, depreciating slightly vis-à-vis the U.S. dollar but appreciating in trade-weighted terms.
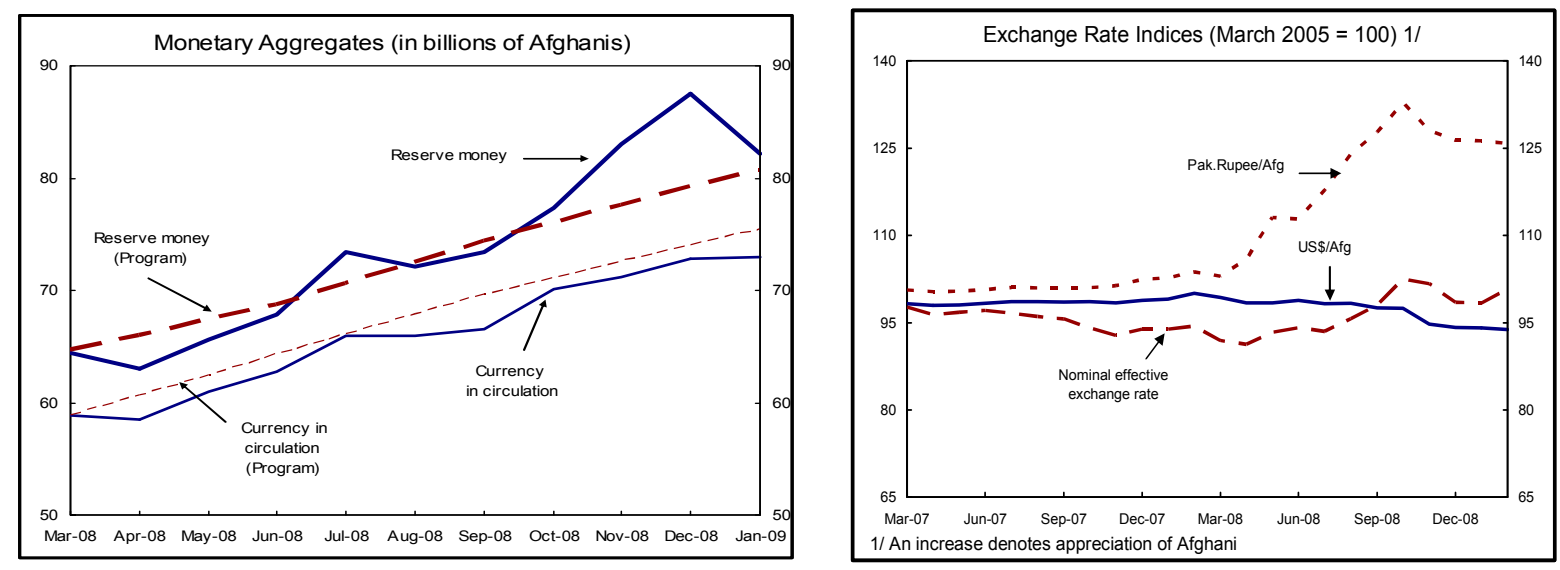

11. Except for some fiscal targets, all other quantitative performance criteria were observed. Reserve accumulation exceeded the program targets, and all the other performance criteria under central bank's responsibility and the debt-related provisions were observed (Table 6). However, the end-September performance criterion on domestic revenues was not observed. Based on preliminary estimates, growing fiscal slippages led to a larger deviation from the end-March 2009 performance criterion on domestic revenues and also resulted in a deviation from the end-March 2009 limit on central bank financing of the government. A new set of targets for June 2009 is proposed below for the Sixth Review.

12. Financial supervision has been strengthened. The central bank intervened in a small insolvent private bank in November 2008 and subsequently tightened the definitions governing reserve requirements to ensure that all banks had sufficient liquidity. In addition, the central bank increased the number of bank supervisors, boosted salaries to encourage retention, separated onsite and offsite functions, and doubled the frequency of onsite examinations. ${ }^{9}$ It also started implementing an action plan developed in September 2008 (structural benchmark)

\footnotetext{
${ }^{8}$ To encourage banks to use the notes to manage liquidity, the central bank reduced the return on overnight deposit facilities compared to the rate on 28-day notes and allowed banks to use the notes as collateral for short-term interbank lending.

${ }^{9}$ The banking system has grown at a fast pace since $2004 / 05$, with assets increasing by about 50 percent per year and rising from 6 percent of GDP to almost 20 percent in 2008/09.
} 
to address weaknesses in the legal framework as identified in a self-assessment of the Basel Core Principles for Effective Banking Supervision.

13. The central bank has been proceeding with internal reforms. Starting in October 2008, monetary data have been compiled from the accounting system in line with technical assistance recommendations. However, the central bank concluded that it was impracticable to produce a monetary survey using a unified timeframe (end-June 2008 structural benchmark) and has subsequently reached understandings with commercial banks to ensure concordance among key data. ${ }^{10}$

14. Reform of public enterprises has been slow. The authorities rejected the sole bid for the privatization of the telephone company, and no subsequent offer has been made. Parliament amended the State-Owned Enterprise Law to stipulate that its approval would in the future be required for any restructuring or liquidation of public enterprises and, citing this provision, objected to the liquidation of a small defunct state-owned bank. The state electricity company exhausted its annual fuel subsidy halfway through the fiscal year. As a result, donors had to redirect an amount equivalent to 0.2 percent of GDP from development assistance to support the company through the remainder of the year. Since then, the Ministry of Finance has prepared a medium-term action plan to reduce electricity subsidies (end-December 2008 structural benchmark).

15. The record of implementation of the structural agenda has been mixed. The audited statements of the state electricity company (end-September 2008 performance criterion) were submitted in March 2009 and four of the nine structural benchmarks scheduled for implementation under the Fifth Review have been delayed (Table 7). Two of the delayed benchmarks have now been implemented, and the remaining two (the external audits of the state fuel company and the review of relations between the government and key public enterprises) have been rescheduled for implementation under the Sixth and Seventh Reviews, respectively. ${ }^{11}$

\section{Policy Discussions}

\section{The authorities concurred on the need to redress policy slippages, ensure} macroeconomic stability, and implement HIPC commitments. The discussions focused on measures to increase revenues and strengthen fiscal governance, to strengthen surveillance of key public enterprises, and to tighten monetary policy to lower inflation.

\footnotetext{
${ }^{10}$ The central bank (which uses the Solar calendar) had intended to produce a monetary survey with a single time frame. Commercial banks use the Gregorian calendar.

${ }^{11}$ The envisaged reduction of the tariff on soft drinks from 40 percent to 20 percent, originally a structural performance criterion for the Sixth Review, has been delayed due to the authorities' concerns about the impact on employment. The authorities have reiterated their commitment to implement this measure in March 2010. Staff proposes that this measure not be retained in structural conditionality under the program.
} 


\section{A. Economic Outlook}

\section{The program for $\mathbf{2 0 0 9 / 1 0}$ envisages a recovery in output and a reduction in}

inflation. The global crisis is not expected to have a large impact on Afghanistan's economy given the country's limited trade and financial links and its reliance on agriculture.

Agriculture is expected to recover with improved weather, but global conditions are expected to cut the growth of industry and services from 13 percent in 2008/09 to 7 percent this year, leading to an overall GDP growth rate of 9 percent. Inflation is targeted to be 6 percent or less. The external current account balance is likely to worsen somewhat due to the impact of global conditions on foreign aid, remittances, and exports. In the medium term, mining and services are projected to increase their contribution to growth.

\section{B. Fiscal Issues}

18. The program targets a lower fiscal deficit in $\mathbf{2 0 0 9 / 1 0}$. Revenues and grants for operating expenditures are projected to increase by 0.6 percent and 0.3 percent of GDP, respectively (Table 4). As operating expenditures are expected to decrease slightly, the deficit including grants is expected to decline from 1.1 percent of GDP in 2008/09 to near zero in 2009/10. Excluding grants, the operating budget deficit would fall from 5.5 percent of GDP to 4.7 percent. $^{12}$

\section{In March 2009, the authorities implemented three prior actions for the} completion of the Fifth Review. These measures and their rationales are as follows: (i) submitting audited financial statements of the state electricity company (to mitigate quasifiscal risks); (ii) amending income tax legislation to introduce the business receipts tax (an embryonic VAT) on imports (to broaden the tax base); and (iii) ensuring that customs officials have access to the state fuel company depots in Hairatan (to improve customs collection and governance) and preparing a report documenting such access.

20. The program envisages additional revenue measures to boost collection and improve administration (Attachment II, paragraphs 17-19). The authorities have recently pursued administrative reforms including personnel changes, which have contributed to a turnaround in revenue performance since December 2008. The additional measures are:

$>\quad$ extending the use of the Automated System for Customs Data (ASYCUDA) modules to Kabul airport and some provinces (benchmark for April 2009); extending the large tax payers offices to two more provinces (staff recommended that the new offices be under the control of headquarters to ensure their success);

\footnotetext{
12 The 2009/10 figures do not include additional military spending of 1.5 percentage points of GDP that is contingent on donor financing. If this increase materializes, the deficit excluding grants could rise to 6.2 percent of GDP. The target date for equating operating expenditures with domestic revenues has slipped to 2014/15, one year later than envisaged at the time of the Fourth Review. The target date could be put back further if the army's size is doubled over the next few years as planned.
} 
preparing an action plan to enhance the responsibility of the tax and customs departments for human and financial resources;

setting up post-clearance audit units at two important border posts (Jalalabad and Mazar-e-Sharif) (benchmark for May 2009) and establishing continuous customs controls at private fuel depots; and

monitoring collection of the business receipts tax on imports (structural benchmark for June 2009).

21. The overall fiscal envelope is contained and spending priorities are in line with the Afghanistan National Development Strategy (ANDS). For 2009/10, the expenditure envelope will be largely determined by domestic revenues and grants, with minimal financing from central bank deposits and foreign loans (Table 4). The main increases in spending are for wages and items related to defense, health, and education. To enhance the capacity to identify and prevent arrears, the authorities intend to focus on cash planning and management through credible spending ceilings and improved accounting and recording.

\section{The authorities agreed on the need to address fiscal risks from key public}

enterprises. Despite several delays, the audits of the state fuel company are expected to be completed by June 2009 and a thorough review of the financial situation of key public enterprises (the state airline Ariana, the state electricity company, Afghan Telecom, and the state fuel company) should be finalized by January 2010. The medium-term goal is to regularize these companies' fiscal relations with the government, adopt modern corporate governance, and proceed with their divestiture, restructuring, or privatization. ${ }^{13}$ The authorities agreed that it would be useful to bring public enterprises under one oversight body in the Ministry of Finance, but indicated that they need more time to consider such a measure. With regard to the new requirement for Parliamentary approval for restructuring or liquidating public enterprises ( $₫ 14)$, the authorities interpreted that defunct state-owned banks are subject to the Commercial Code, not the amended State-Owned Enterprise Law. Staff recommended that this requirement be clarified to avoid undue delays in the process.

\section{Monetary and Financial Sector Issues}

23. The monetary program aims at reducing inflation to 6 percent by March 2010. Having accommodated the impact of high food and fuel prices in 2008, the central bank intends to lock in the recent decrease in world food and fuel prices by cutting monetary growth. In 2009/10, reserve money is expected to grow by 17 percent, compared to 35 percent in 2008/09. To attain its monetary targets, the central bank plans to intensify the use of capital notes and continue promoting the secondary market for these notes.

\footnotetext{
${ }^{13}$ Separately, the state electricity company will be subject to stricter monitoring of its operations and financial flows through an improved reporting system, and the two state-owned banks will be expected to improve their CAMEL ratings (Capital adequacy, Asset quality, Management, Earnings, and Liquidity) and implement pending recommendations from central bank supervisors.
} 


\section{The program targets a modest buildup of international reserves and assumes} a managed floating exchange rate. Net international reserves are programmed to increase by US $\$ 200$ million, allowing import coverage to remain at a comfortable level of about 11 months of imports. The central bank envisages conducting foreign exchange market interventions mainly to smooth market volatility, but not to resist underlying trends.

25. The central bank plans to proceed with financial sector reforms. Staff noted that the rapid increase of the banking system warrants continuing vigilance and further strengthening of the central bank's supervisory capacity. The authorities concurred and pointed out that they intend to automate offsite examinations through information technology and to supplement the examination of banks with low CAMEL ratings with targeted examinations. There are also plans to introduce a credit bureau and a collateral registry (with World Bank assistance), and a limited deposit insurance scheme geared toward small depositors.

\section{External Sector and Debt}

26. The external current account balance is projected to worsen in 2009/10.

Projections for exports and transit trade have been lowered in line with the deteriorating global outlook. In the medium term, the current account deficit is not likely to decline, although import demand should shift to investment goods as security improves and the new copper mine (Aynak) is set up to begin operations (Table 8).

27. The HIPC completion point could take place during the fourth quarter of 2009. This will require the completion of the Sixth Review and meeting the HIPC triggers. ${ }^{14}$ Afghanistan's main bilateral creditors (the United States, Germany, and Russia) have committed to provide 100 percent debt relief, which is beyond what is required under the Enhanced HIPC initiative. ${ }^{15}$ The authorities have expressed their commitment to implement the HIPC triggers in the remainder of this year (Box 1).

\section{Program Monitoring}

\section{The authorities are requesting an extension of the PRGF arrangement through}

March 2010. The extension will allow the authorities more time to implement pending reforms and to demonstrate their continued commitment to economic stability. The authorities are also requesting the inclusion of a Seventh Review based on end-December 2009 data and other measures. To accommodate the additional program

\footnotetext{
${ }^{14}$ The completion triggers were specified in Islamic Republic of Afghanistan: Enhanced Heavily Indebted Poor Countries Initiative - Decision Point Document and Debt Sustainability Analysis, IMF Country Report No. 07/253, July 2007.

${ }^{15}$ Following the completion point, Afghanistan would be eligible for debt relief under the Multilateral Debt Relief Initiative (MDRI) from the International Development Agency (there is no eligible debt to the Fund). Debts to remaining foreign creditors are small, and the authorities have sought debt forgiveness in light of Afghanistan's HIPC status.
} 
Box 1. Status of HIPC Completion Point Triggers

$\begin{array}{ll}\text { Trigger Status } & \end{array}$

Satisfactory ANDS implementation

Establishment of external debt database and publication of three consecutive quarterly reports in a timely manner

Alignment of public spending priorities with the ANDS

Production of medium-term fiscal framework consistent with PRGF and ANDS priorities

Tracking poverty-related spending over a period of at least six months preceding the completion point

Submission to Parliament for the year immediately preceding the completion point of (i) the annual core budget audit report and (ii) a report on the resolution of important issues identified by the external auditor

Design pension reform for public employees and the military

Restructure the Ministries of Agriculture, Education, Public Health, and Rural Development to strengthen core functions and service delivery mechanisms

Develop and adopt regulations and guidelines for minerals extraction and establish a fully operational hydrocarbon/mining cadastre

Annually publish government and third-party data on the quantity of health and education services
In progress. A report on implementation is expected in June

Met. Regular publication of the reports is expected to continue.

Met. The budget department has been reorganized, and seven pilot ministries presented their 2008/09 budgets broadly aligned with the ANDS. The mechanism should continue to be refined.

Met.

Met. The Chart of Accounts has been modified, allowing the seven pilot ministries to track expenditure according to the defined programs. The bridging of expenditure data should be refined to provide greater detail on the expenditure outcomes in the nonpilot ministries.

In progress. The annual audit report was submitted to Parliament on September 20, 2008. The second report has not yet been submitted.

In progress. The Cabinet adopted a pension policy strategy in early-2008, and project preparation for implementation of the strategy has been initiated.

In progress.

In progress. The Minerals Law and a revised version of the Hydrocarbon Law have been approved. Preparation of implementing regulations is in progress. Establishment of the cadastre has been initiated. In March 2009, the authorities announced that Afghanistan will join the Extractive Industries Transparency Initiative.

In progress. Report plans for health have not yet been developed. For education, reports on school grant usage have been produced.

Sources: Afghan authorities and World Bank and Fund staff.

disbursement, the seventh disbursement, linked to the Sixth Review, will be split in two. An extension request for the Paris Club arrangement (for which a current PRGF arrangement is required) will be made following the completion of the Fifth Review.

29. The Sixth and Seventh Reviews will track progress in the program's pressing priorities, namely fiscal sustainability, revenue collection and related governance issues, and fiscal risks from public enterprises. Structural benchmarks have been set accordingly, as specified in the Supplementary Memorandum of Economic and Financial Policies and in 
the Technical Memorandum of Understanding (Attachment 2, Table 2, and Attachment III). Quantitative performance criteria for the Sixth Review will be based on end-June 2009 data (Attachment II, Table 1). Staff is also proposing quantitative performance criteria for the Seventh Review with an end-December 2009 test date (Attachment II, Table 1). The structural benchmarks for the Seventh Review will be revisited at the time of the Sixth Review (Attachment II, Table 2).

\section{Staff Appraisal}

30. Against the backdrop of serious security challenges, Afghanistan's performance under the program was mixed. On one hand, the authorities have pursued prudent financial sector policies and are resolved to lower inflation after a sharp increase in food and fuel prices. On the other hand, revenue performance, a key feature of the PRGF arrangement, was weaker than expected and a number of structural reforms were delayed. The envisaged date when domestic revenues would cover operating expenditures has been pushed back by one year.

31. The economic program for 2009/10 aims at consolidating achievements, redressing slippages, and proceeding with much-needed reforms. The conduct of monetary policy and recent inflation data are encouraging, but the success of the program will also hinge on adherence to the fiscal targets and pressing ahead with reforms to boost revenues and reduce fiscal risks from public enterprises. Staff therefore urges the authorities to adhere to their goal of a balanced budget including grants and welcomes their commitment to cut expenditures should revenues fall short of programmed levels. Furthermore, the staff encourages the authorities to undertake all necessary actions to ensure a sustained increase in revenues and to use the medium-term fiscal framework as a planning tool. Since substantial outlays will be required in the next decade to rebuild the country and meet poverty-reduction goals, increased revenue collection will be critical to reduce aid dependency.

32. Staff urges the authorities to advance reforms in revenue administration and deal with pervasive corruption. Key reforms comprise collecting the business receipts tax on imports, establishing effective customs controls on the state fuel company and on private fuel depots, and further automating operations and expanding post-clearance audits at customs. Staff welcomes the extension of the large taxpayers' office to more provinces and encourages the authorities to proceed with their plan to establish medium taxpayers' offices. It will also be important for the revenue departments to enjoy more autonomy in financial and human resources management.

33. A cautious approach to public spending and cash management to avoid arrears will also be critical. Increased military spending is essential in the current environment, and the authorities should ensure that these increases are fully financed by donors. The authorities should be similarly cautious in implementing civil service salary reforms to prevent unsustainable increases in operating expenditures. To avoid expenditure arrears, staff urges the authorities to build up discretionary cash balances of at least two weeks of operating expenditures or 0.5 percent of GDP.

34. Monetary policy appropriately focuses on disinflation. Staff concurs with the authorities' objective of reducing inflation to 6 percent or less. Inflation trends should be 
closely monitored and monetary policy adjusted accordingly if inflation does not remain anchored at low levels. At the same time, the authorities should maintain a managed floating exchange rate policy to buffer the economy from real shocks and support the implementation of monetary policy. Monetary policy operations will also be enhanced by the development of the secondary market for capital notes.

35. The central bank is expected to step up the monitoring of commercial banks. Afghanistan's linkages to global financial markets are believed to be fairly limited, nonperforming loan ratios remain low, and the intervention of a small bank in November 2008 went smoothly. However, the continuing rapid expansion of commercial banks and lending highlights the need to strengthen supervision and fully enforce prudential norms, especially on state-owned banks.

36. Public enterprises continue to pose fiscal risks and should be closely monitored. Financial data on the largest public enterprises remain inadequate, governance problems abound, and progress to reform these companies has been scant. Staff urges the authorities to complete the audits of the state fuel company and privatize it without delay and to strengthen control of the operations of the state electricity company. There is also a need to bring all public enterprises under one oversight body in the Ministry of Finance, finalize the review of fiscal relations of key public enterprises; prepare business plans; and move ahead with their restructuring, divestiture, or privatization. Lastly, staff urges the authorities to seek a clarification of the requirement under the amended State-Owned Enterprise Law that Parliamentary approval is needed for the privatization of public enterprises and liquidation of state-owned banks.

37. The program is exposed to various risks, most notably a volatile security situation or political turmoil as the presidential election approaches. Notwithstanding these risks, it remains important to redouble efforts to maintain fiscal discipline, take a strong stance against corruption, and implement the envisaged reforms. Successful implementation of the program and of other HIPC conditions will allow Afghanistan to obtain near-100 percent debt relief under the HIPC Initiative and the MDRI.

38. Based on the implementation of the agreed prior actions, the recent turnaround in revenue collection, and the strength of the 2009/10 program, staff supports the waivers of nonobservance of performance criteria and the completion of the Fifth Review. Staff also supports the extension of the arrangement and the rephasing of disbursements. 
Figure 1. Islamic Republic of Afghanistan: Output, Inflation, and External Sector Developments
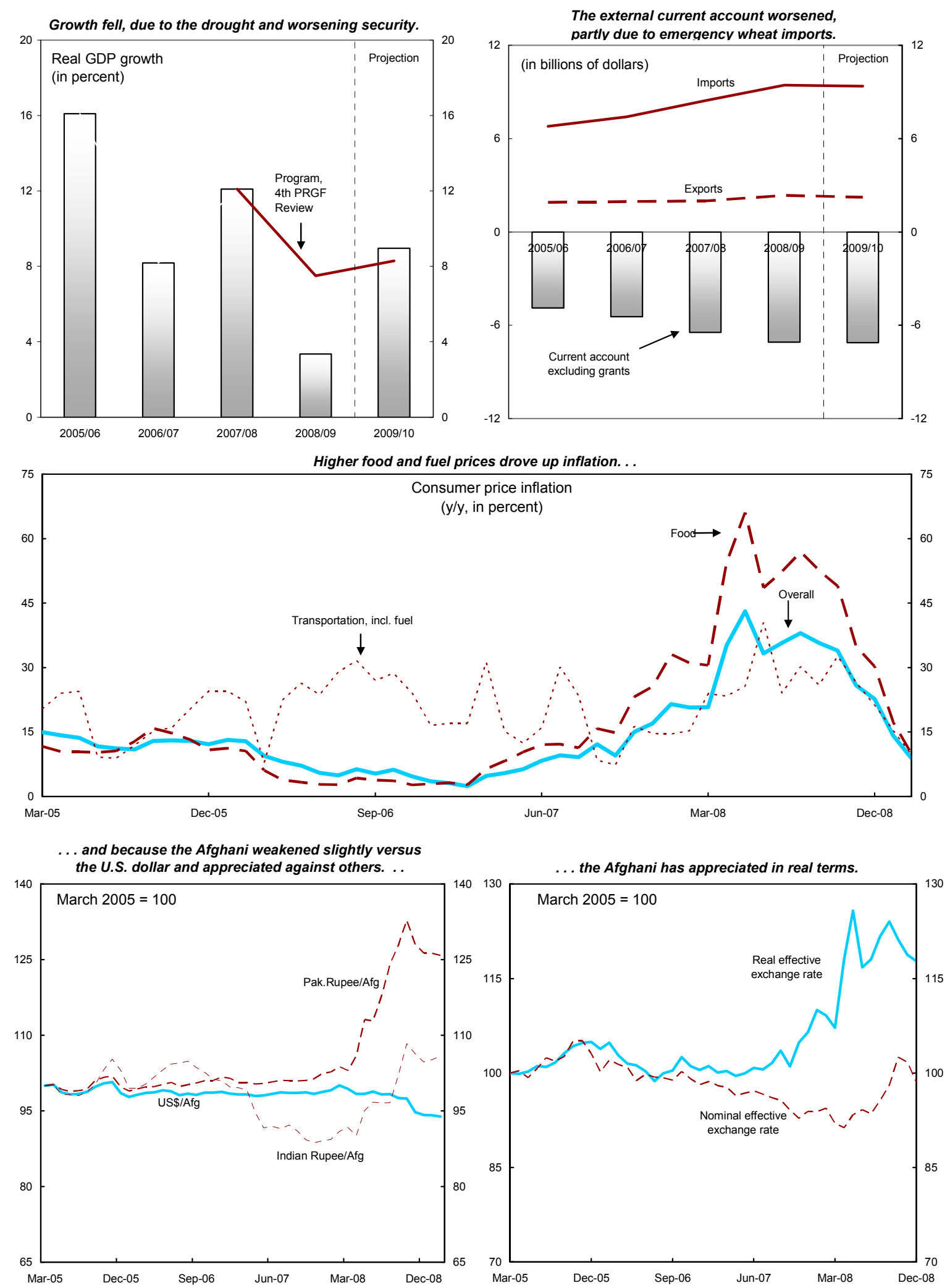

Source: Da Afghanistan Bank, Central Statistical Office; and Fund staff estimates. 
Figure 2. Islamic Republic of Afghanistan: Fiscal Developments
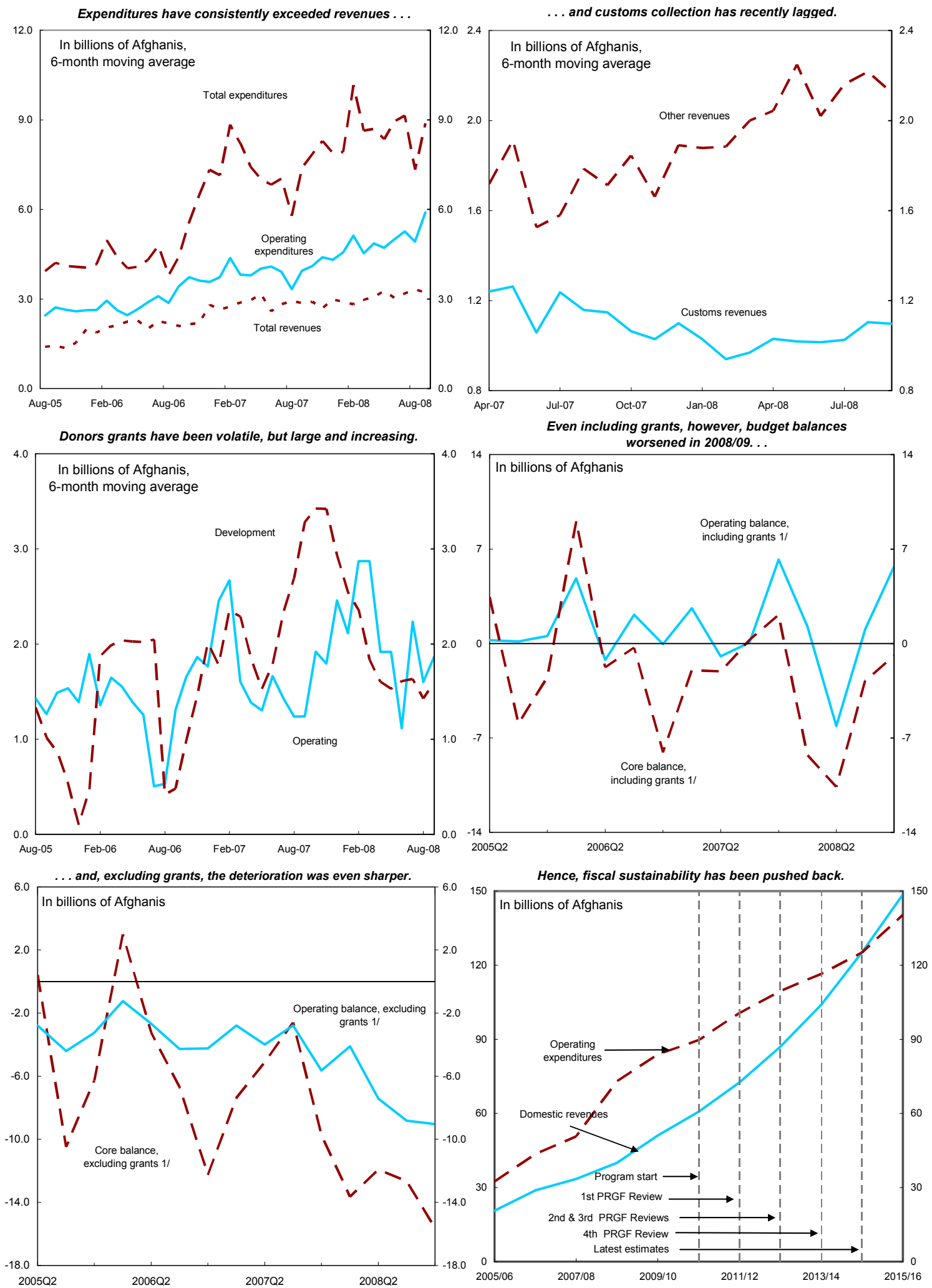

Source: Ministry of Finance; and Fund staff estimates.

$1 /$ The operating balance includes only operating expenditures; the core balance includes both operating and development expenditures.

2/ Fiscal sustainability is defined in the program as a zero operating balance excluding grants. 
Figure 3. Islamic Republic of Afghanistan: Monetary and Financial Developments
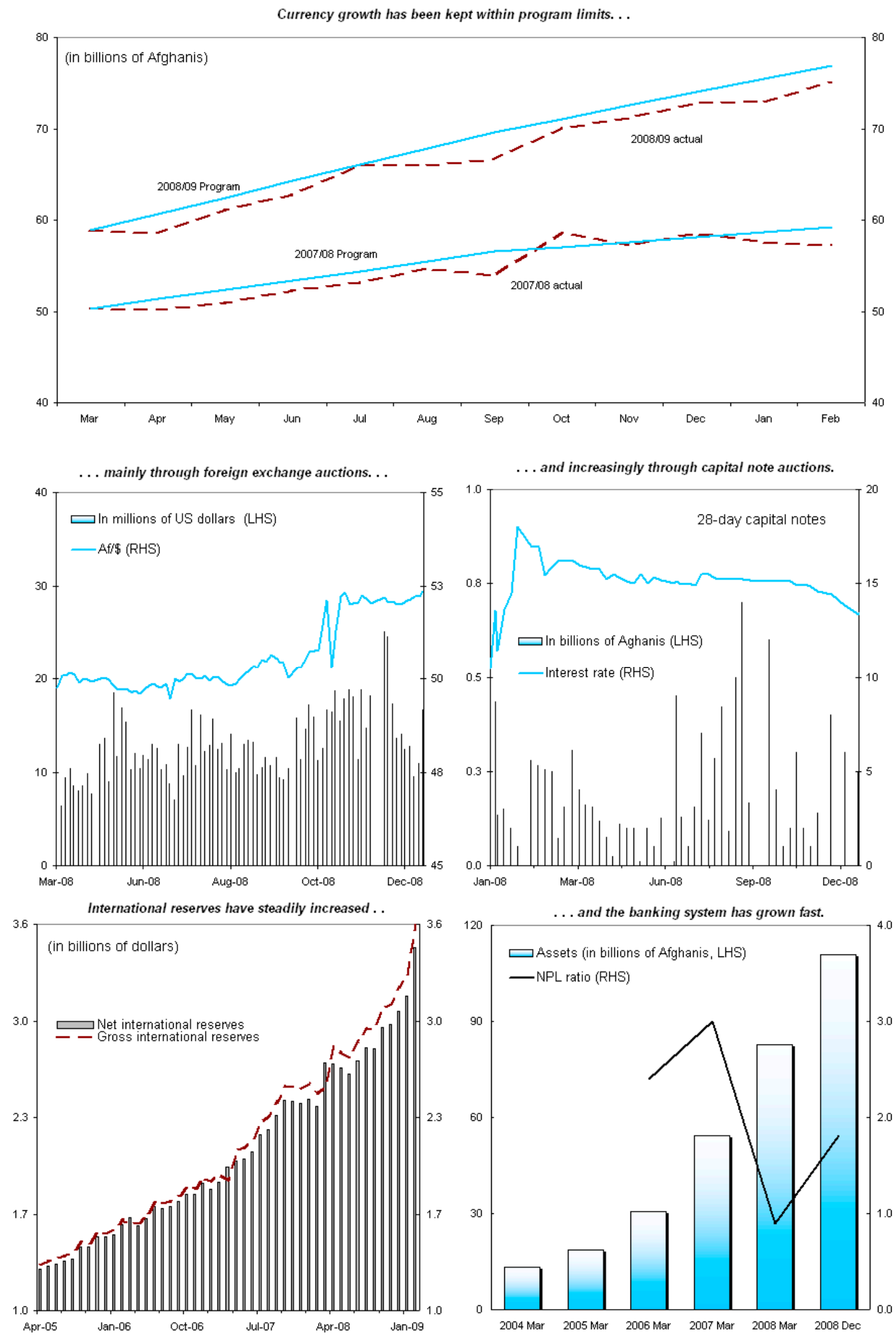

Source: Da Afahanistan Bank (DAB), and Fund staff estimates. 
Table 1. Islamic Republic of Afghanistan: Schedule of Reviews and Disbursements

\begin{tabular}{|c|c|c|c|}
\hline \multirow[b]{2}{*}{ Date } & \multicolumn{2}{|c|}{ Amount of Disbursement } & \multirow[b]{2}{*}{ Conditions } \\
\hline & Millions of SDRs & Percent of Quota 1/ & \\
\hline June 2006 & 13.2 & 8.2 & Approval of arrangement 2/ \\
\hline March 2007 & 11.3 & 7.0 & $\begin{array}{l}\text { First review and September } 2006 \\
\text { performance criteria 3/ }\end{array}$ \\
\hline July 2007 & 11.3 & 7.0 & $\begin{array}{l}\text { Second review and March } 2007 \\
\text { performance criteria 4/ }\end{array}$ \\
\hline February 2008 & 11.3 & 7.0 & $\begin{array}{l}\text { Third review and September } 2007 \\
\text { performance criteria 5/ }\end{array}$ \\
\hline June 2008 & 11.3 & 7.0 & $\begin{array}{l}\text { Fourth review and March } 2008 \\
\text { performance criteria 6/ }\end{array}$ \\
\hline April 2009 & 11.3 & 7.0 & $\begin{array}{l}\text { Fifth review and September } 2008 \\
\text { performance criteria } 7 /\end{array}$ \\
\hline September 2009 & 5.7 & 3.5 & $\begin{array}{l}\text { Sixth review and June } 2009 \\
\text { performance criteria 8/ }\end{array}$ \\
\hline March 2010 & 5.7 & 3.5 & $\begin{array}{l}\text { Seventh review and December } 2009 \\
\text { performance criteria 9/ }\end{array}$ \\
\hline Total & 81.0 & 50.0 & \\
\hline
\end{tabular}

Source: Fund staff estimates.

$1 /$ Does not sum due to rounding.

2/ Due to domestic legal obstacles, the first disbursement was drawn with a delay, on January 19, 2007.

3/ The First Review was completed on March 7, 2007 and the second disbursement was drawn on March $29,2007$.

4/ The Second Review was completed on July 9, 2007 and the third disbursement was drawn on July 23, 2007.

5/ The Third Review was completed on February 14, 2008 and the fourth disbursement was drawn on February $28,2008$.

6/ The Fourth Review was completed on July 7, 2008 and the fifth disbursement was drawn on July 15, 2008.

7/ The board meeting on the Fifth Review is tentatively scheduled for April 2009.

8/ The board meeting on the Sixth Review is expected in September 2009, along with the HIPC completion point.

9/ The board meeting on the Seventh Review is expected in March 2010. 
Table 2. Islamic Republic of Afghanistan: Selected Economic Indicators, 2005/06-2009/10

(Quota: SDR 161.9 million)

(Population: 26.7 million; 2006/07)

(Per capita GDP: US\$289; 2006/07)

(Poverty rate: n.a.)

(Main export: carpets, US\$186 million; 2006/07)

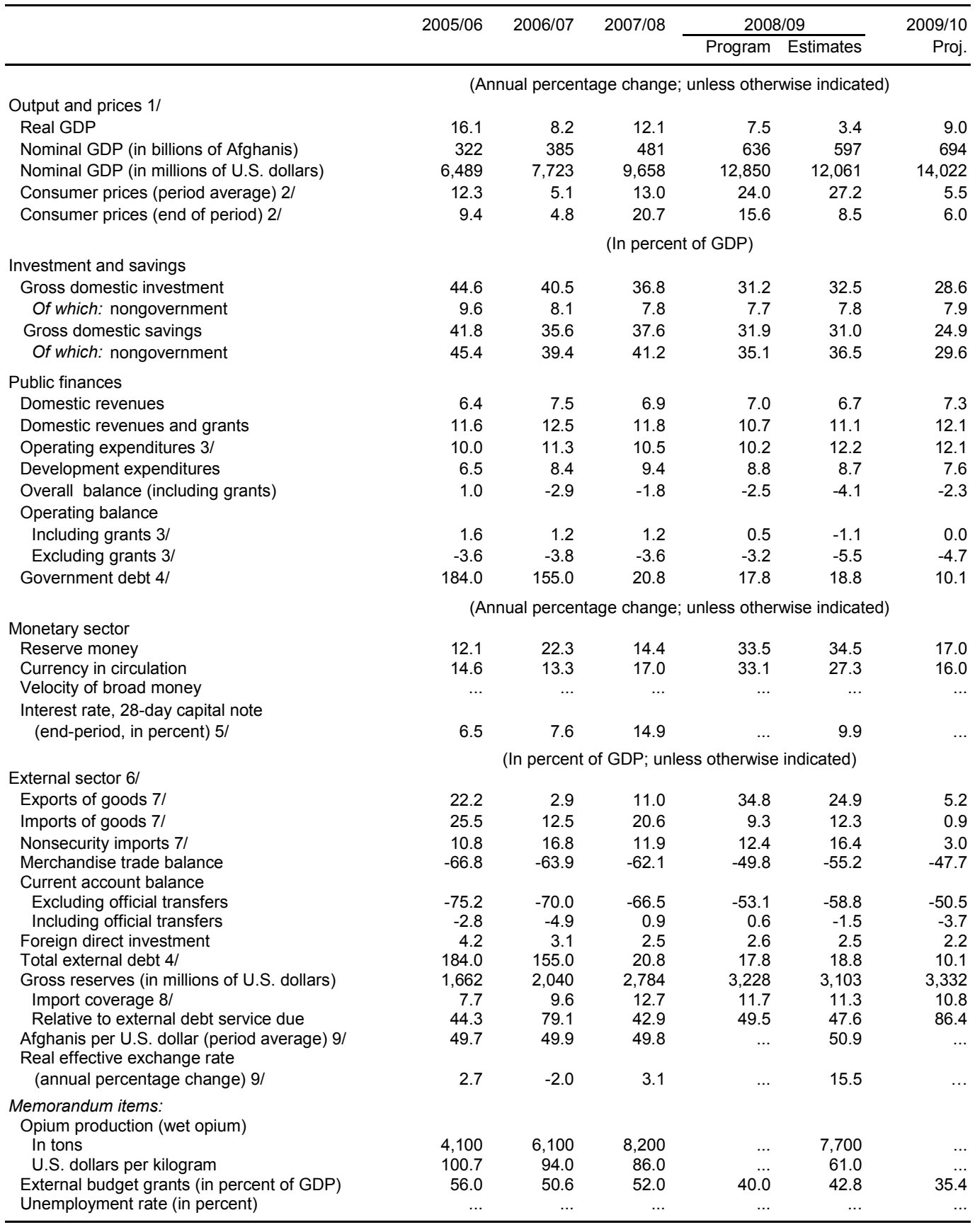

Sources: Afghan authorities; United Nations Office on Drugs and Crime; and Fund staff estimates and projections.

$1 /$ Excluding the drug economy.

2/ Kabul only.

3/ Excluding development spending and externally financed development expenditures.

4/ After HIPC and MDRI debt relief as well as debt relief beyond HIPC relief from Paris Club creditors. Debt also includes obligations to the IMF.

5/ The 2008/09 number is for March 10, 2009.

$6 /$ Includes official recorded exports plus staff estimates of smuggling; excludes reexports.

7/ In U.S. dollars, percentage change.

8/ In months of next year's imports of goods and services, excluding imports for reexports and duty-free imports by donors.

9/ The latest actual data: February 2009 for the Afghani per U.S. dollar and December 2008 for real effective exchange rate.

10/ The 2008/09 figure is for February 2009. 
Table 3. Islamic Republic of Afghanistan: Balance of Payments, 2005/06-2009/10

\begin{tabular}{|c|c|c|c|c|c|}
\hline & $2005 / 06$ & $2006 / 07$ & $2007 / 08$ & $\begin{array}{r}2008 / 09 \\
\text { Est. }\end{array}$ & $\begin{array}{r}2009 / 10 \\
\text { Proj. }\end{array}$ \\
\hline & \multicolumn{5}{|c|}{ (In millions of U.S. dollars; unless otherwise indicated) } \\
\hline Current account (including grants) & -182 & -379 & 85 & -181 & -519 \\
\hline Current account (excluding grants) & $-4,880$ & $-5,406$ & $-6,425$ & $-7,087$ & $-7,083$ \\
\hline Trade balance & $-4,335$ & $-4,933$ & $-6,002$ & $-6,658$ & $-6,681$ \\
\hline Exports of goods (f.o.b.) 1/ & 1,795 & 1,811 & 1,835 & 2,145 & 2,012 \\
\hline Official exports & 386 & 416 & 482 & 603 & 658 \\
\hline Unofficial exports (smuggling and transit trade) & 1,409 & 1,394 & 1,352 & 1,542 & 1,354 \\
\hline Imports of goods (f.o.b.) & $-6,130$ & $-6,744$ & $-7,837$ & $-8,803$ & $-8,694$ \\
\hline Official imports & $-5,482$ & $-6,049$ & $-7,246$ & $-8,273$ & $-8,093$ \\
\hline Of which: Duty free & $-3,258$ & $-3,579$ & $-4,685$ & $-5,455$ & $-4,897$ \\
\hline Smuggling & -648 & -694 & -590 & -529 & -601 \\
\hline Services and income, net & -545 & -473 & -423 & -429 & -401 \\
\hline Of which: Interest due 2/ 3/ & -21 & -17 & -61 & -61 & -35 \\
\hline Current transfers & 4,698 & 5,027 & 6,510 & 6,906 & 6,564 \\
\hline Public & 4,361 & 4,625 & 6,068 & 6,381 & 6,117 \\
\hline Private (including through licensed money changers) & 337 & 401 & 441 & 525 & 447 \\
\hline Capital and financial account & 357 & 194 & 25 & 121 & -762 \\
\hline Capital transfers & 0 & 0 & 0 & 0 & 0 \\
\hline Debt forgiveness $3 /$ & 0 & 0 & 0 & 0 & $-1,061$ \\
\hline Foreign direct investment & 271 & 238 & 243 & 300 & 312 \\
\hline Official loans (net) & 85 & 155 & 129 & 121 & 153 \\
\hline Disbursement & 102 & 164 & 133 & 125 & 156 \\
\hline Amortization due 2/3/ & -16 & -9 & -4 & -4 & -4 \\
\hline Other items (net) & $\ldots$ & -198 & -347 & -300 & -165 \\
\hline Errors and omissions (including short-term capital) & 223 & 405 & 370 & 420 & 0 \\
\hline Overall balance & 398 & 220 & 480 & 360 & $-1,281$ \\
\hline Financing & -398 & -220 & -480 & -360 & 1,247 \\
\hline Changes in reserve assets of the central bank & -426 & -255 & -571 & -430 & 157 \\
\hline Use of Fund resources (net) & 0 & 20 & 51 & 17 & 0 \\
\hline Exceptional financing & 28 & 15 & 40 & 53 & 1,090 \\
\hline Arrears 4/ & -1 & -132 & $-11,007$ & -84 & 0 \\
\hline Debt rescheduling, of which: $5 /$ & 0 & 117 & 777 & 80 & 29 \\
\hline Capitalization of interest & 0 & 4 & 47 & 48 & 24 \\
\hline Multilateral HIPC assistance & 0 & 0 & 3 & 5 & 5 \\
\hline Debt forgiveness, of which: $3 /$ & 29 & 30 & 10,270 & 56 & 1,061 \\
\hline HIPC & 0 & 0 & 0 & 0 & 442 \\
\hline MDRI & 0 & 0 & 0 & 0 & 35 \\
\hline Financing gap & 0 & 0 & 0 & 0 & -34 \\
\hline Identified financing (provisional) & 0 & 0 & 0 & 0 & 34 \\
\hline Of which: IMF PRGF & 0 & 0 & 0 & 0 & 34 \\
\hline Remaining gap & 0 & 0 & 0 & 0 & 0 \\
\hline \multicolumn{6}{|l|}{ Memorandum items: } \\
\hline Gross international reserves & 1,662 & 2,040 & 2,784 & 3,103 & 3,332 \\
\hline (In months of imports) 6/ & 7.7 & 9.6 & 12.7 & 11.3 & 10.8 \\
\hline (Relative to external debt service due) & 44.3 & 79.1 & 42.9 & 47.6 & 86.4 \\
\hline (Relative to commercial bank foreign currency liabilities) & 2.6 & 2.2 & 2.2 & 2.0 & 1.9 \\
\hline \multicolumn{6}{|l|}{ Current account balance (percent of GDP) } \\
\hline Including grants & -2.8 & -4.9 & 0.9 & -1.5 & -3.7 \\
\hline Excluding grants & -75.2 & -70.0 & -66.5 & -58.8 & -50.5 \\
\hline Total debt service (percent of exports, excluding reexports) 7/ & 5.7 & 1.6 & 1.1 & 1.3 & 0.9 \\
\hline Total debt stock (percent of GDP) $7 /$ & 184.0 & 155.0 & 20.8 & 18.8 & 10.1 \\
\hline
\end{tabular}

Sources: Afghan authorities; and Fund staff estimates and projections.

1/ Excludes opium exports and, due to limited data availability, flows associated with U.S. Army and most ISAF activities. 2/ Debt service projections are based on the total stock of external debt (including estimates of unverified arrears). Interest on overdue obligations represents estimates by Fund staff.

3/ Assumes that Afghanistan will reach the HIPC completion point. Paris Club creditors are assumed to provide 100 percent debt stock reduction.

4/ Arrears shown represent Fund staff estimates of debt service due, but not paid, on estimated overdue obligations.

5/ Debt rescheduling includes the capitalization of interset falling due to Paris Club creditors until the completion point, interim assistance from multilateral creditors, and HIPC debt relief from multilateral creditors after the completion point. 6/ Excluding imports for reexports and duty free imports by donors.

7/ After HIPC and MDRI relief as well as debt relief beyond HIPC from Paris Club creditors. Debt includes obligations to the IMF. The debt stock includes the capitalization of interest to Paris Club creditors until the completion point of the enhanced HIPC initiative. 
Table 4a. Islamic Republic of Afghanistan: Core Budget, 2005/06-2009/10 1/

\begin{tabular}{|c|c|c|c|c|c|c|}
\hline & \multirow[t]{2}{*}{$2005 / 06$} & \multirow{2}{*}{\multicolumn{2}{|c|}{$2006 / 072007 / 08$}} & \multicolumn{2}{|c|}{$2008 / 09$} & \multirow{2}{*}{$\begin{array}{r}2009 / 10 \\
\text { Proj }\end{array}$} \\
\hline & & & & Prog. & Est. & \\
\hline & \multicolumn{6}{|c|}{ (In billions of Afghanis) } \\
\hline Domestic revenues & 20.7 & 28.8 & 33.4 & 44.5 & 40.0 & 51.0 \\
\hline Tax revenues & 14.0 & 21.9 & 25.0 & 34.7 & 30.0 & 39.4 \\
\hline Income, profits, and capital gains & 2.6 & 6.7 & 7.6 & 9.8 & 8.5 & 10.1 \\
\hline International trade and transactions (customs) & 9.4 & 12.0 & 12.9 & 17.8 & 13.8 & 17.8 \\
\hline Goods and services & 1.8 & 2.1 & 3.5 & 5.8 & 6.3 & 9.9 \\
\hline Other & 0.2 & 1.1 & 1.0 & 1.3 & 1.3 & 1.6 \\
\hline Nontax revenues & 6.6 & 6.9 & 8.4 & 9.8 & 10.0 & 11.6 \\
\hline Grants to operating budget $2 /$ & 16.7 & 19.2 & 23.1 & 23.5 & 26.4 & 32.9 \\
\hline Afghanistan Reconstruction Trust Fund (recurrent window) & 12.6 & 14.9 & 14.5 & 13.7 & 15.7 & 13.0 \\
\hline Law and Order Trust Fund for Afghanistan & 4.1 & 3.7 & 6.9 & 7.6 & 8.0 & 13.7 \\
\hline Other grants & 0.1 & 0.5 & 1.7 & 2.2 & 2.8 & 6.2 \\
\hline Grants to development budget & 19.3 & 16.6 & 30.3 & 37.2 & 33.9 & 36.4 \\
\hline Total expenditures & 53.4 & 75.7 & 95.7 & 121.3 & 125.1 & 136.7 \\
\hline Operating expenditures & 32.3 & 43.4 & 50.7 & 65.0 & 73.0 & 83.8 \\
\hline Wages and salaries & 20.4 & 26.5 & 33.6 & 44.8 & 48.9 & 56.6 \\
\hline Purchase of goods and services & 6.7 & 10.2 & 11.0 & 11.7 & 15.4 & 18.7 \\
\hline Transfers, subsidies, and other & 0.5 & 2.2 & 1.7 & 3.9 & 3.9 & 3.2 \\
\hline Pensions & 1.5 & 2.5 & 2.8 & 3.2 & 2.9 & 3.3 \\
\hline Capital expenditures & 3.1 & 2.0 & 1.4 & 1.0 & 1.6 & 1.6 \\
\hline Interest & 0.1 & 0.2 & 0.1 & 0.3 & 0.3 & 0.4 \\
\hline Development expenditures 3/ & 21.1 & 32.3 & 45.0 & 56.3 & 52.1 & 52.8 \\
\hline Security & 1.1 & 1.4 & 1.5 & 1.8 & 1.0 & 0.3 \\
\hline Governance, rule of law, and human rights & 0.6 & 0.8 & 0.7 & 0.9 & 1.6 & 1.2 \\
\hline Infrastructure and natural resources & 6.2 & 13.0 & 19.3 & 24.2 & 21.8 & 21.3 \\
\hline Education & 1.0 & 1.8 & 3.2 & 4.0 & 4.1 & 5.9 \\
\hline Health & 1.0 & 2.1 & 2.2 & 2.7 & 2.5 & 5.3 \\
\hline Agriculture and rural development & 10.2 & 12.0 & 15.8 & 19.7 & 18.1 & 13.2 \\
\hline Social protection & 0.0 & 0.0 & 0.1 & 0.1 & 0.1 & 0.8 \\
\hline Economic governance and private sector development & 1.0 & 1.1 & 2.3 & 2.9 & 2.9 & 4.8 \\
\hline Operating balance including grants & 5.0 & 4.6 & 5.9 & 3.0 & -6.5 & 0.1 \\
\hline Operating balance excluding grants & -11.7 & -14.7 & -17.2 & -20.5 & -33.0 & -32.8 \\
\hline Overall budget balance including grants & 3.2 & -11.1 & -8.8 & -16.2 & -24.7 & -16.3 \\
\hline Float and adjustment $4 /$ & -2.0 & 1.2 & 9.0 & 0.0 & 0.0 & 0.0 \\
\hline Sale of nonfinancial assets $5 /$ & 2.0 & 2.3 & 0.1 & 9.9 & 4.0 & 0.0 \\
\hline Financing & -3.2 & 7.5 & -0.2 & 6.3 & 20.7 & 16.3 \\
\hline External loans (net) & 5.3 & 6.1 & 6.1 & 6.0 & 6.0 & 7.9 \\
\hline Domestic (net) 6/ & -8.5 & 1.4 & -6.3 & 0.3 & 14.7 & 8.4 \\
\hline \multicolumn{7}{|l|}{ Memorandum items: } \\
\hline Discretionary cash balances $7 /$ & $\ldots$ & $\ldots$ & 4.0 & 16.9 & 1.4 & 1.5 \\
\hline Fuel subsidy to DABM/S (state electricity company) & $\ldots$ & 2.2 & 1.7 & 2.0 & 3.4 & 2.1 \\
\hline External budget $8 /$ & 180.6 & 195.0 & 250.2 & 254.2 & 255.6 & 245.9 \\
\hline Of which: security & 81.2 & 80.5 & 124.6 & 123.8 & 123.8 & 122.3 \\
\hline Contingent military spending $9 /$ & $\ldots$ & $\ldots$ & $\ldots$ & $\ldots$ & $\ldots$ & 10.4 \\
\hline
\end{tabular}


Table 4b. Islamic Republic of Afghanistan: Core Budget, 2005/06-2009/10 1/

\begin{tabular}{|c|c|c|c|c|c|c|}
\hline & \multirow[t]{2}{*}{$2005 / 06$} & \multirow{2}{*}{\multicolumn{2}{|c|}{$2006 / 072007 / 08$}} & \multicolumn{2}{|c|}{$2008 / 09$} & \multirow{2}{*}{$\begin{array}{r}2009 / 10 \\
\text { Proj. }\end{array}$} \\
\hline & & & & Prog. & Est. & \\
\hline & \multicolumn{6}{|c|}{ (In percent of GDP) } \\
\hline Domestic revenues & 6.4 & 7.5 & 6.9 & 7.0 & 6.7 & 7.3 \\
\hline Tax revenues & 4.4 & 5.7 & 5.2 & 5.4 & 5.0 & 5.7 \\
\hline Income, profits, and capital gains & 0.8 & 1.7 & 1.6 & 1.5 & 1.4 & 1.5 \\
\hline International trade and transactions (customs) & 2.9 & 3.1 & 2.7 & 2.8 & 2.3 & 2.6 \\
\hline Goods and services & 0.5 & 0.5 & 0.7 & 0.9 & 1.1 & 1.4 \\
\hline Others & 0.1 & 0.3 & 0.2 & 0.2 & 0.2 & 0.2 \\
\hline Nontax revenues & 2.1 & 1.8 & 1.8 & 1.5 & 1.7 & 1.7 \\
\hline Grants to operating budget $2 /$ & 5.2 & 5.0 & 4.8 & 3.7 & 4.4 & 4.7 \\
\hline Afghanistan Reconstruction Trust Fund (recurrent window) & 3.9 & 3.9 & 3.0 & 2.2 & 2.6 & 1.9 \\
\hline Law and Order Trust Fund for Afghanistan & 1.3 & 1.0 & 1.4 & 1.2 & 1.3 & 2.0 \\
\hline Other grants & 0.0 & 0.1 & 0.4 & 0.4 & 0.5 & 0.9 \\
\hline Grants to development budget & 6.0 & 4.3 & 6.3 & 5.8 & 5.7 & 5.2 \\
\hline Total expenditures & 16.6 & 19.6 & 19.9 & 19.1 & 20.9 & 19.7 \\
\hline Operating expenditures & 10.0 & 11.3 & 10.5 & 10.2 & 12.2 & 12.1 \\
\hline Wages and salaries & 6.3 & 6.9 & 7.0 & 7.0 & 8.2 & 8.1 \\
\hline Purchase of goods and services & 2.1 & 2.6 & 2.3 & 1.8 & 2.6 & 2.7 \\
\hline Transfers, subsidies, and other & 0.2 & 0.6 & 0.4 & 0.6 & 0.7 & 0.5 \\
\hline Pensions & 0.5 & 0.6 & 0.6 & 0.5 & 0.5 & 0.5 \\
\hline Capital expenditures & 0.9 & 0.5 & 0.3 & 0.2 & 0.3 & 0.2 \\
\hline Interest & 0.0 & 0.0 & 0.0 & 0.0 & 0.0 & 0.1 \\
\hline Development expenditures $3 /$ & 6.5 & 8.4 & 9.4 & 8.8 & 8.7 & 7.6 \\
\hline Security & 0.3 & 0.4 & 0.3 & 0.3 & 0.2 & 0.0 \\
\hline Governance, rule of law, and human rights & 0.2 & 0.2 & 0.2 & 0.1 & 0.3 & 0.2 \\
\hline Infrastructure and natural resources & 1.9 & 3.4 & 4.0 & 3.8 & 3.7 & 3.1 \\
\hline Education & 0.3 & 0.5 & 0.7 & 0.6 & 0.7 & 0.9 \\
\hline Health & 0.3 & 0.5 & 0.5 & 0.4 & 0.4 & 0.8 \\
\hline Agriculture and rural development & 3.2 & 3.1 & 3.3 & 3.1 & 3.0 & 1.9 \\
\hline Social protection & 0.0 & 0.0 & 0.0 & 0.0 & 0.0 & 0.1 \\
\hline Economic governance and private sector development & 0.3 & 0.3 & 0.5 & 0.4 & 0.5 & 0.7 \\
\hline Operating balance including grants & 1.6 & 1.2 & 1.2 & 0.5 & -1.1 & 0.0 \\
\hline Operating balance excluding grants & -3.6 & -3.8 & -3.6 & -3.2 & -5.5 & -4.7 \\
\hline Overall budget balance including grants & 1.0 & -2.9 & -1.8 & -2.5 & -4.1 & -2.3 \\
\hline Float and adjustment 4/ & -0.6 & 0.3 & 1.9 & 0.0 & 0.0 & 0.0 \\
\hline Sale of nonfinancial assets $5 /$ & 0.6 & 0.6 & 0.0 & 1.6 & 0.7 & 0.0 \\
\hline Financing & -1.0 & 2.0 & 0.0 & 1.0 & 3.5 & 2.3 \\
\hline External loans (net) & 1.7 & 1.6 & 1.3 & 0.9 & 1.0 & 1.1 \\
\hline Domestic (net) 6/ & -2.6 & 0.4 & -1.3 & 0.0 & 2.5 & 1.2 \\
\hline \multicolumn{7}{|l|}{ Memorandum items: } \\
\hline Discretionary cash balances $7 /$ & $\ldots$ & $\ldots$ & 0.8 & 2.6 & 0.2 & 0.2 \\
\hline Fuel subsidy to DABM/S (state electricity company) & $\ldots$ & 0.6 & 0.4 & 0.3 & 0.6 & 0.3 \\
\hline External budget $8 /$ & 56.0 & 50.6 & 52.0 & 40.0 & 42.8 & 35.4 \\
\hline Of which: security & 25.2 & 20.9 & 25.9 & 19.5 & 20.7 & 17.6 \\
\hline Contingent military spending $9 /$ & & & & & & 1.5 \\
\hline Nominal GDP (in billions Afs) & 322.2 & 385.5 & 481.2 & 636.2 & 597.2 & 694.3 \\
\hline
\end{tabular}

Sources: Ministry of Finance; Da Afghanistan Bank; and Fund staff estimates and projections.

$1 /$ Reflects the accounts of the central government, and does not include spending on public goods financed by donors outside the budget.

2/ Funding for operating budget from multidonor trust funds: The Afghanistan Reconstruction Trust Fund (ARTF; recurrent window) and the Law and Order Trust Fund for Afghanistan (LOTFA).

3/ Interim Afghanistan National Development Strategy programs.

4/ Variation between the fiscal position recorded at the Ministry of Finance and the central bank. This discrepancy is partly due to the difference ("float") between checks issued and checks cashed.

$5 /$ In 2005/06 and 2006/07 includes US $\$ 40$ million receipt from sale of telecommunications spectrum bandwidth and late overflight paymens. From 2007/08 includes sale of land and buildings and privatization receipts.

6/ Net change in government deposits with the central bank (excluding provincial branch balances). A positive sign corresponds to a decline in balances.

$7 /$ Government deposits at the central bank excluding earmarked grants.

8/ Estimated direct expenditures by donors on public projects not included in the core budget.

9/ To be applied as an adjustors to the program depending on the Afghan National Army exceeding 109,000. 
Table 5. Islamic Republic of Afghanistan: Monetary Program (Central Bank), 2007/08-2009/10

\begin{tabular}{|c|c|c|c|c|c|c|c|c|c|c|c|c|c|}
\hline & \multirow{3}{*}{$\begin{array}{r}2007 / 081 / \\
\text { Mar. } 19\end{array}$} & \multirow{3}{*}{$\begin{array}{r}\text { 2008/09 2/ } \\
\text { Jun. } 20\end{array}$} & \multirow{2}{*}{\multicolumn{2}{|c|}{$\begin{array}{l}2008 / 09 \\
\text { Sep. } 21\end{array}$}} & \multirow{2}{*}{\multicolumn{2}{|c|}{$\frac{2008 / 09}{\text { Dec. } 20}$}} & \multirow{2}{*}{\multicolumn{2}{|c|}{$\begin{array}{l}2008 / 09 \\
\text { Mar. } 20 \\
\end{array}$}} & \multirow{3}{*}{$\begin{array}{l}2008 / 09 \\
\text { Mar. } 20 \\
\end{array}$} & \multicolumn{4}{|c|}{$2009 / 10$} \\
\hline & & & & & & & & & & Jun. 20 & Sep. 20 & Dec. 20 & Mar. 20 \\
\hline & & & Prog. & Actual 21 & Prog. & $\overline{\text { Actual } 21}$ & Prog. & Est. 2l & & \multicolumn{2}{|c|}{ Program $3 /$} & & \\
\hline & \multicolumn{13}{|c|}{ (In billions of Afghanis, unless otherwise noted) } \\
\hline Net foreign assets & 143.0 & 137.2 & 149.1 & 148.2 & 162.1 & 161.0 & 163.8 & 160.6 & 165.5 & 168.5 & 172.0 & 174.0 & 175.6 \\
\hline Foreign assets & 148.0 & 142.3 & 155.0 & 153.1 & 168.1 & 165.8 & 170.7 & 165.4 & 170.2 & 174.1 & 178.1 & 180.1 & 182.1 \\
\hline Foreign exchange reserves & 137.9 & 133.9 & 144.6 & 143.7 & 157.5 & 157.4 & 159.8 & 157.4 & 161.8 & 165.7 & 169.7 & 171.7 & 173.7 \\
\hline Other foreign assets & 10.1 & 8.4 & 10.4 & 9.4 & 10.6 & 8.4 & 10.8 & 8.0 & 8.4 & 8.4 & 8.4 & 8.4 & 8.4 \\
\hline Foreign liabilities & -5.0 & -5.0 & -6.0 & -4.8 & -6.0 & -4.8 & -6.9 & -4.8 & -4.7 & -5.7 & -6.1 & -6.1 & -6.6 \\
\hline Net domestic assets & -78.6 & -69.3 & -72.4 & -74.8 & -80.5 & -73.4 & -77.8 & -73.9 & -78.8 & -78.6 & -77.5 & -76.0 & -74.1 \\
\hline Domestic assets & -44.2 & -34.2 & -38.8 & -38.5 & -46.9 & -39.7 & -44.2 & -40.2 & -41.4 & -41.2 & -40.1 & -38.6 & -36.7 \\
\hline Net claims on general government & -34.9 & -25.1 & -29.0 & -28.0 & -37.0 & -26.3 & -34.0 & -20.2 & -21.4 & -21.2 & -21.4 & -18.4 & -13.0 \\
\hline Gross claims on government & 3.8 & 3.8 & 4.8 & 4.7 & 4.8 & 4.7 & 5.7 & 4.7 & 4.7 & 5.6 & 6.1 & 6.1 & 6.5 \\
\hline Domestic currency deposits & -7.9 & -4.3 & -8.0 & -5.8 & -9.2 & -3.4 & -9.6 & -2.5 & -2.5 & -3.4 & -3.8 & -3.4 & -3.0 \\
\hline Foreign currency deposits & -30.9 & -24.5 & -25.8 & -26.8 & -32.6 & -27.6 & -30.1 & -22.4 & -23.6 & -23.5 & -23.6 & -21.1 & -16.5 \\
\hline Other claims (including capital notes) & -9.3 & -9.1 & -9.7 & -10.5 & -9.9 & -13.4 & -10.2 & -20.0 & -20.0 & -19.9 & -18.8 & -20.2 & -23.7 \\
\hline Of which: capital notes & -4.7 & -5.1 & -5.8 & -7.2 & -6.4 & -8.8 & -7.0 & -15.7 & -15.7 & -15.7 & -14.6 & -16.2 & -19.8 \\
\hline Other items net & -34.3 & -35.2 & -33.6 & -36.3 & -33.6 & -33.7 & -33.6 & -33.7 & -37.4 & -37.4 & -37.4 & -37.4 & -37.4 \\
\hline Reserve money & 64.4 & 67.9 & 76.7 & 73.4 & 81.6 & 87.6 & 86.0 & 86.7 & 86.7 & 89.9 & 94.5 & 98.0 & 101.4 \\
\hline Currency in circulation & 58.9 & 62.8 & 69.6 & 66.6 & 74.0 & 72.8 & 78.4 & 75.0 & 75.0 & 77.9 & 82.1 & 84.6 & 87.0 \\
\hline Banknotes and coins issued & 66.7 & 69.8 & 79.2 & 73.0 & 84.3 & 77.2 & 89.4 & 79.5 & 79.5 & 82.5 & 87.0 & 89.7 & 92.2 \\
\hline Less: cash holdings & 7.8 & 7.0 & 9.6 & 6.4 & 10.3 & 4.4 & 11.0 & 4.5 & 4.5 & 4.7 & 4.9 & 5.1 & 5.2 \\
\hline Bank deposits with DAB & 5.5 & 5.1 & 7.0 & 6.8 & 7.6 & 14.7 & 7.6 & 11.7 & 11.7 & 12.0 & 12.4 & 13.4 & 14.4 \\
\hline \multicolumn{14}{|l|}{ Memorandum items: } \\
\hline \multicolumn{14}{|l|}{ Reserve money } \\
\hline Year-to-date percentage change & 14.4 & 5.4 & 19.0 & 13.9 & 26.6 & 35.9 & 33.5 & 34.5 & 34.5 & 3.7 & 9.0 & 13.1 & 17.0 \\
\hline Year-on-year percentage change & 14.4 & 21.7 & 32.8 & 27.1 & 29.0 & 38.5 & 33.5 & 34.5 & 34.5 & 32.4 & 28.7 & 11.9 & 17.0 \\
\hline \multicolumn{14}{|l|}{ Currency in circulation } \\
\hline Year-to-date percentage change & 17.0 & 6.6 & 18.2 & 13.0 & 25.7 & 23.6 & 33.1 & 27.3 & 27.3 & 3.9 & 9.5 & 12.9 & 16.0 \\
\hline Year-on-year percentage change & 17.0 & 20.1 & 29.0 & 23.4 & 26.4 & 24.4 & 33.1 & 27.3 & 27.3 & 24.0 & 23.3 & 16.2 & 16.0 \\
\hline \multicolumn{14}{|l|}{ Gross international reserves } \\
\hline At market rates; in millions of US dollars & 2,784 & 2,666 & & 2,751 & & 2,851 & & & & & & & \\
\hline At program rates; in millions of US dollars & 2,784 & 2,704 & 2,921 & 2,901 & 3,180 & 3,178 & 3,228 & 3,178 & 3,103 & 3,179 & 3,255 & 3,294 & 3,332 \\
\hline \multicolumn{14}{|l|}{ Net international reserves } \\
\hline (at program rates; in millions of US dollars) & 2,669 & 2,595 & 2,762 & 2,766 & 2,999 & 3,016 & 3,007 & 3,022 & 2,955 & 3,013 & 3,082 & 3,122 & 3,152 \\
\hline Consumer prices in Kabul & & & & & & & & & & & & & \\
\hline (year-on-year change) & 20.7 & 33.3 & $\ldots$ & 35.7 & $\ldots$ & 22.7 & $\ldots$ & 8.5 & 8.5 & 7.5 & 5.0 & 6.0 & 6.0 \\
\hline Interest rates on 28-day capital notes 4 / & 15.2 & 15.0 & $\ldots$ & 14.5 & $\ldots$ & 13.0 & $\ldots$ & & & & & & \\
\hline Af/US $\$$ (end of period) exchange rate & 49.52 & 49.82 & 49.52 & 50.48 & 49.52 & 52.14 & 49.52 & 49.52 & 52.14 & 52.14 & 52.14 & 52.14 & 52.14 \\
\hline
\end{tabular}

Sources: Da Afghanistan Bank (DAB) and Central Statistics Office; and Fund staff estimates and projections.

$1 /$ Foreign currency-denominated components evaluated using the actual exchange rates at date indicated.

2/ Foreign currency-denominated components evaluated using applicable program exchange rates as of March 19, 2008.

3/ Foreign currency-denominated components evaluated using applicable program exchange rates as of December 20, 2008.

4/ Weighted average of bid rates; 30-day notes until March 2006. 
Table 6. Islamic Republic of Afghanistan: Quantitative Performance Criteria and Indicative Targets under the PRGF Arrangement, 2008/09 1/

\begin{tabular}{|c|c|c|c|c|c|c|c|c|c|c|c|c|c|}
\hline & \multirow{3}{*}{$\frac{\text { Mar. } 19,2008}{\text { Stocks }}$} & \multicolumn{3}{|c|}{ Jun. 19, 2008} & \multicolumn{3}{|c|}{ Sept. 21, 2008} & \multicolumn{3}{|c|}{ Dec. 20, 2008} & \multicolumn{3}{|c|}{ Mar. 20, 2009} \\
\hline & & \multicolumn{2}{|c|}{ Indicative Targets } & \multirow[t]{2}{*}{ Actual } & \multicolumn{2}{|c|}{ Performance Criteria } & \multirow[t]{2}{*}{ Actual } & \multicolumn{2}{|c|}{ Indicative Targets } & \multirow[t]{2}{*}{ Actual } & \multicolumn{2}{|c|}{ Performance Criteria } & \multirow[t]{2}{*}{ Estimate } \\
\hline & & $\begin{array}{l}\text { Cntry. Rept. } \\
08 / 229\end{array}$ & Adjusted & & $\begin{array}{l}\text { Cntry. Rept. } \\
08 / 229\end{array}$ & Adjusted & & $\begin{array}{l}\text { Cntry. Rept. } \\
08 / 229\end{array}$ & Adjusted & & $\begin{array}{l}\text { Cntry. Rept. } \\
08 / 229\end{array}$ & Adjusted & \\
\hline & \multicolumn{13}{|c|}{ (In billions of Afghanis) } \\
\hline Floor on fiscal revenues of the government & $\ldots$ & 8.5 & $\ldots$ & 7.9 & 18.4 & $\ldots$ & 18.2 & 30.0 & $\ldots$ & 27.2 & 44.5 & $\ldots$ & 40.0 \\
\hline Ceiling on currency in circulation & 58.9 & 5.4 & $\ldots$ & 3.9 & 10.7 & $\ldots$ & 7.7 & 15.1 & $\ldots$ & 13.9 & 19.5 & $\ldots$ & 16.1 \\
\hline $\begin{array}{l}\text { Ceiling on net central bank financing of the } \\
\text { government }\end{array}$ & -34.9 & 1.4 & 10.2 & 9.9 & 5.3 & 12.4 & 7.0 & -2.7 & 12.5 & 8.6 & 0.0 & 8.1 & 14.7 \\
\hline $\begin{array}{l}\text { Indicative target (ceiling) on the operating budget } \\
\text { deficit of the government, excluding grants }\end{array}$ & $\ldots$ & 4.5 & $\ldots$ & 4.2 & 10.9 & $\ldots$ & 11.3 & 15.5 & $\ldots$ & 22.1 & 20.5 & 22.5 & 33.0 \\
\hline \multirow[t]{2}{*}{ Indicative target (ceiling) on reserve money } & 64.4 & 6.3 & $\ldots$ & 3.5 & 12.2 & $\ldots$ & 9.0 & 17.1 & $\ldots$ & 23.1 & 21.6 & $\ldots$ & 22.2 \\
\hline & \multicolumn{13}{|c|}{ (In millions of U.S. dollars) } \\
\hline Floor on net international reserves of $D A B$ & $2,669.3$ & 72.4 & -105.4 & -74.1 & 93.0 & -50.7 & 96.8 & 329.6 & 23.7 & 346.5 & 338.1 & 174.1 & 352.2 \\
\hline $\begin{array}{l}\text { Ceiling on contracting or guaranteeing new medium- } \\
\text { and long-term nonconcessional external debt by the } \\
\text { government and DAB 2/ }\end{array}$ & $\ldots$ & 0.0 & $\ldots$ & 0.0 & 0.0 & $\ldots$ & 0.0 & 0.0 & $\ldots$ & 0.0 & 0.0 & $\ldots$ & 0.0 \\
\hline $\begin{array}{l}\text { Ceiling on short-term external debt owed or } \\
\text { guaranteed by the government or DAB } 2 /\end{array}$ & $\ldots$ & 0.0 & $\ldots$ & 0.0 & 0.0 & $\ldots$ & 0.0 & 0.0 & $\ldots$ & 0.0 & 0.0 & $\ldots$ & 0.0 \\
\hline $\begin{array}{l}\text { New external payments arrears, excluding interest } \\
\text { on preexisting arrears } 2 /\end{array}$ & $\ldots$ & 0.0 & & 0.0 & 0.0 & $\ldots$ & 0.0 & 0.0 & $\ldots$ & 0.0 & 0.0 & $\ldots$ & 0.0 \\
\hline \multirow[t]{2}{*}{$\begin{array}{l}\text { Ceiling on lending from state-owned banks to SOES } \\
\text { in need of restructuring or government guaranteeing } \\
\text { borrowing by these SOEs } 2 /\end{array}$} & $\ldots$ & 0.0 & $\ldots$ & 0.0 & 0.0 & $\ldots$ & 0.0 & 0.0 & $\ldots$ & 0.0 & 0.0 & $\ldots$ & 0.0 \\
\hline & \multicolumn{13}{|c|}{ (In billions of Afghanis) } \\
\hline $\begin{array}{l}\text { Memorandum items: } \\
\text { Operating budget deficit of the government, } \\
\text { including grants }\end{array}$ & $\ldots$ & -0.2 & $\ldots$ & 4.2 & 0.3 & $\ldots$ & 1.7 & -1.0 & $\ldots$ & 51 & -30 & . & 65 \\
\hline Reference projections for the adjustors & & Program & Actual & Deviation & Program & Actual & Deviation & Program & Actual & Deviation & Program & Estimate & Deviation \\
\hline Core budget development spending & $\ldots$ & 7.0 & 3.2 & -3.9 & 21.1 & 14.5 & -6.7 & 35.2 & 24.6 & -10.6 & 56.3 & 52.1 & -4.2 \\
\hline $\begin{array}{l}\text { External financing of the core budget and sale or } \\
\text { transfers of nonfinancial assets }\end{array}$ & $\ldots$ & 10.1 & 1.3 & -8.8 & 26.8 & 19.6 & -7.1 & 53.3 & 38.2 & -15.1 & 76.6 & 70.4 & -6.2 \\
\hline Additional security spending $3 /$ & $\ldots$ & $\ldots$ & $\ldots$ & $\ldots$ & $\ldots$ & $\ldots$ & $\ldots$ & $\ldots$ & . & $\ldots$ & 0.0 & 1.9 & 1.9 \\
\hline
\end{tabular}

Source: Fund staffe

1/ The performance criteria and indicative targets envisaged under the program, and their adjustors, are defined in Attachment III of Country Report 08/229.

2/ These performance criteria apply on a continuous basis.

3/ Defined in Paragraph 21 of Attachment III of Country Report 08/229. 
Table 7. Islamic Republic of Afghanistan: Structural Conditionality under the PRGF Arrangement June-December 2008

\section{Fifth Review}

Performance Criteria

Submission to Parliament of the core budget's audited financial statement for 2007/08.

Submission of Da Afghanistan Breshna Moassessa

(DABM) audited financial statements for 2006/07-07/08

Benchmarks

The central bank will produce the Monetary Survey for 2007/08 using a consistent time frame (Solar or Gregorian) for the entire banking system.

Notify traders of the expiration of exemption from the general income tax regime and inform that they will be expected to file and pay their tax liabilities for 2008/09 according to the general provisions applying to corporate income tax payers.

Develop an action plan to address the gaps in the legal framework based on the weaknesses identified in the central bank's self-assessment against the Basel

Principles.

Create an electronic registry of capital notes at the central bank.

Roll out the Large Taxpayers Office to Herat, Jalalabad, and Mazar-e-Sharif.

Amend income tax legislation, including introduction of the business receipts tax on imports.

Publish external audit report of the Fuel and Liquid Gas

Enterprise (FLGE).

Review fiscal relations between the government and Afghan Telecom, Ariana, DABM/S, and FLGE.

The Ministry of Finance will conclude a comprehensive agreement with DABM on a medium-term plan to reduce subsidies.
Target Dates

September 21, 2008

September 30, 2008

June 30, 2008

July 31,2008

September 30, 2008

September 30, 2008

September 30, 2008

November 30, 2008

November 30, 2008

December 31, 2008

December 31, 2008
Status

Met

Not met (prior action for completion of Fifth Review)

Not met, but understandings reached between the central bank and commercial banks on concordance for major items.

Met

Met

Met

Met

Not met (prior action for completion of Fifth Review)

Not met (will be benchmark for Sixth Review)

Not met (will be benchmark for Seventh Review)

Not met on time. The agreement was signed on February 22, 2009, by Ministers of Finance, Energy and Water, and Economy 
Table 8. Islamic Republic of Afghanistan: Medium-Term Macroeconomic Framework, 2008/09-2013/14

\begin{tabular}{|c|c|c|c|c|c|c|}
\hline & $2008 / 09$ & $2009 / 10$ & $2010 / 11$ & $2011 / 12$ & $2012 / 13$ & $2013 / 14$ \\
\hline & Est. & \multicolumn{5}{|c|}{ Projections } \\
\hline & \multicolumn{6}{|c|}{ (Annual percentage change; unless otherwise indicated) } \\
\hline \multicolumn{7}{|l|}{ Output and prices $1 /$} \\
\hline Real GDP & 3.4 & 9.0 & 7.0 & 8.1 & 8.8 & 9.2 \\
\hline Nominal GDP (in millions of U.S. dollars) & 12,061 & 14,022 & 15,327 & 16,877 & 18,718 & 20,848 \\
\hline GDP per capita (in U.S. dollars) & 429 & 485 & 517 & 554 & 599 & 649 \\
\hline \multirow[t]{2}{*}{ Consumer prices (period average) 2/ } & 27.2 & 5.5 & 5.4 & 5.0 & 5.0 & 5.0 \\
\hline & \multicolumn{6}{|c|}{ (In percent of GDP, unless otherwise indicated) } \\
\hline \multicolumn{7}{|l|}{ Investment and savings } \\
\hline Gross investment & 32.5 & 28.6 & 29.4 & 30.0 & 31.8 & 29.8 \\
\hline Of which: nongovernment & 7.8 & 7.9 & 10.1 & 11.9 & 14.9 & 15.2 \\
\hline Gross savings & 31.0 & 24.9 & 24.7 & 24.9 & 25.1 & 22.1 \\
\hline Of which: nongovernment & 36.5 & 29.6 & 28.5 & 28.2 & 27.4 & 20.9 \\
\hline \multicolumn{7}{|l|}{ Public finances } \\
\hline Domestic revenues & 6.7 & 7.3 & 7.8 & 8.3 & 8.8 & 9.3 \\
\hline Domestic revenues and grants & 11.1 & 12.1 & 11.7 & 11.4 & 11.0 & 11.0 \\
\hline Operating expenditures $3 /$ & 12.2 & 12.1 & 11.6 & 11.6 & 11.1 & 10.4 \\
\hline Development expenditures & 8.7 & 7.6 & 7.5 & 7.3 & 7.1 & 6.9 \\
\hline Overall balance (including grants) & -4.1 & -2.3 & -2.1 & -1.5 & -1.4 & -1.3 \\
\hline \multicolumn{7}{|l|}{ Operating balance } \\
\hline Including grants 3/ & -1.1 & 0.0 & 0.1 & -0.2 & -0.1 & 0.5 \\
\hline \multirow[t]{2}{*}{ Excluding grants $3 /$} & -5.5 & -4.7 & -3.7 & -3.2 & -2.3 & -1.1 \\
\hline & \multicolumn{6}{|c|}{ (In percent of GDP, unless otherwise indicated) } \\
\hline \multicolumn{7}{|l|}{ External sector 4/ } \\
\hline Exports of goods 5/ & 24.9 & 5.2 & 8.5 & 8.7 & 12.7 & 15.3 \\
\hline Imports of goods $5 /$ & 12.3 & 0.9 & 2.9 & 5.9 & 6.8 & 0.6 \\
\hline Nonsecurity imports $5 /$ & 16.4 & 3.0 & 5.0 & 7.5 & 8.5 & 2.9 \\
\hline Merchandise trade balance & -55.2 & -47.7 & -44.5 & -42.7 & -40.8 & -36.1 \\
\hline \multicolumn{7}{|l|}{ Current account balance } \\
\hline Excluding official transfers & -58.8 & -50.5 & -47.3 & -45.5 & -43.8 & -39.1 \\
\hline Including official transfers & -1.5 & -3.7 & -4.7 & -5.1 & -6.7 & -7.8 \\
\hline Foreign direct investment & 2.5 & 2.2 & 3.6 & 4.2 & 5.9 & 6.0 \\
\hline External debt 6/ & 18.8 & 10.1 & 10.7 & 10.9 & 11.0 & 11.1 \\
\hline Gross reserves (in millions of U.S. dollars) & 3,103 & 3,332 & 3,237 & 3,169 & 3,114 & 2,911 \\
\hline Import coverage $7 /$ & 11.3 & 10.8 & 9.8 & 8.7 & 7.9 & 6.7 \\
\hline Relative to external debt service due & 47.6 & 86.4 & 195.8 & 168.1 & 99.6 & 60.3 \\
\hline \multicolumn{7}{|l|}{ Memorandum items: } \\
\hline External budget grants (in percent of GDP) & 42.8 & 35.4 & 31.8 & 29.0 & 26.3 & 21.5 \\
\hline
\end{tabular}

Sources: Afghan authorities; and Fund staff estimates and projections.

$1 /$ Excluding the drug economy.

2/ Kabul only.

3/ Mainly current expenditures.

4/ Includes official recorded exports plus staff estimates of smuggling; excludes reexports.

5/ In U.S. dollars, percentage change.

6/ After HIPC and MDRI debt relief, as well as debt relief beyond HIPC relief from Paris Club creditors. The debt stock also includes obligations to the IMF.

7/ In months of next year's imports of goods and services, excluding imports for reexports and dutyfree imports. 
Table 9. Islamic Republic of Afghanistan: External Financing Requirement and Sources, 2006/07-2013/14

\begin{tabular}{|c|c|c|c|c|c|c|c|c|}
\hline & $2006 / 07$ & $2007 / 08$ & $2008 / 09$ & $2009 / 10$ & 2010/11 & $2011 / 12$ & $2012 / 13$ & 2013/14 \\
\hline & & & Est. & \multicolumn{5}{|c|}{ Projections } \\
\hline & \multicolumn{8}{|c|}{ (In millions of U.S. dollars) } \\
\hline I. Total financing requirement & 5,801 & 18,007 & 7,605 & 7,991 & 7,164 & 7,617 & 8,148 & 7,972 \\
\hline Current account (excluding grants) & 5,406 & 6,425 & 7,087 & 7,083 & 7,256 & 7,682 & 8,191 & 8,148 \\
\hline Amortization & 9 & 4 & 4 & 4 & 3 & 4 & 13 & 26 \\
\hline Of which: IMF & 0 & 0 & 0 & 0 & 0 & 0 & 8 & 13 \\
\hline Prepayment of debt & 0 & 0 & 0 & 1,061 & 0 & 0 & 0 & 0 \\
\hline Change in reserves (increase $=+$ ) & 255 & 571 & 430 & -157 & -95 & -68 & -55 & -202 \\
\hline Reduction in arrears & 132 & 11,007 & 84 & 0 & 0 & 0 & 0 & 0 \\
\hline II. Available financing & 5,801 & 18,007 & 7,605 & 7,957 & 7,164 & 7,617 & 8,148 & 7,972 \\
\hline Current transfers & 5,027 & 6,510 & 6,906 & 6,564 & 6,539 & 6,824 & 6,937 & 6,530 \\
\hline Foreign direct investment & 238 & 243 & 300 & 312 & 549 & 712 & 1,100 & 1,259 \\
\hline Short-term private financing flows & -198 & -347 & -300 & -165 & -158 & -133 & -126 & -109 \\
\hline Official medium- and long-term loans & 164 & 133 & 125 & 156 & 230 & 210 & 230 & 280 \\
\hline IMF disbursements & 20 & 51 & 17 & 0 & 0 & 0 & 0 & 0 \\
\hline Debt forgiveness & 30 & 10,270 & 56 & 1,061 & 0 & 0 & 0 & 0 \\
\hline Debt rescheduling & 117 & 777 & 80 & 29 & 5 & 5 & 7 & 12 \\
\hline Other & 405 & 370 & 420 & 0 & 0 & 0 & 0 & 0 \\
\hline Required financing (I-II) & 0 & 0 & 0 & 34 & 0 & 0 & 0 & 0 \\
\hline Financing gap & 0 & 0 & 0 & 34 & 0 & 0 & 0 & 0 \\
\hline Identified financing (provisional) & 0 & 0 & 0 & 34 & 0 & 0 & 0 & 0 \\
\hline Of which: IMF PRGF & 0 & 0 & 0 & 34 & 0 & 0 & 0 & 0 \\
\hline Remaining gap & 0 & 0 & 0 & 0 & 0 & 0 & 0 & 0 \\
\hline
\end{tabular}

Sources: Afghan authorities; and Fund staff estimates and projections. 
Table 10. Islamic Republic of Afghanistan: Projected Payments to the Fund as of February 28, 2009

\begin{tabular}{|c|c|c|c|c|c|c|c|c|c|c|}
\hline & $20081 /$ & 2009 & 2010 & 2011 & 2012 & 2013 & 2014 & 2015 & Beyond & Total \\
\hline & \multicolumn{10}{|c|}{ (In millions of SDRs) } \\
\hline \multicolumn{11}{|l|}{$\begin{array}{l}\text { Obligations from existing drawings } \\
\text { 1. Principal }\end{array}$} \\
\hline GRA repurchases & 0.0 & 0.0 & 0.0 & 0.0 & 0.0 & 0.0 & 0.0 & 0.0 & 0.0 & 0.0 \\
\hline PRGF repayments & 0.0 & 0.0 & 0.0 & 0.0 & 2.5 & 8.3 & 11.7 & 11.7 & 24.3 & 58.4 \\
\hline \multicolumn{11}{|l|}{ 2. Charges and interest $2 /$} \\
\hline PRGF interest & 0.3 & 0.3 & 0.3 & 0.3 & 0.3 & 0.3 & 0.2 & 0.1 & 0.0 & 2.1 \\
\hline SDR assessments & 0.0 & 0.0 & 0.0 & 0.0 & 0.0 & 0.0 & 0.0 & 0.0 & 0.0 & 0.0 \\
\hline SDR charges & 0.2 & 0.7 & 0.7 & 0.7 & 0.7 & 0.7 & 0.7 & 0.7 & 5.3 & 10.2 \\
\hline Total obligations & 0.4 & 1.0 & 1.0 & 1.0 & 3.4 & 9.2 & 12.6 & 12.5 & 29.6 & 70.8 \\
\hline (percent of quota) & 0.3 & 0.6 & 0.6 & 0.6 & 2.1 & 5.7 & 7.8 & 7.7 & 18.3 & 43.7 \\
\hline \multicolumn{11}{|l|}{$\begin{array}{l}\text { Obligations from prospective drawings } 3 / \\
\text { 1. Principal }\end{array}$} \\
\hline PRGF repayments & 0.0 & 0.0 & 0.0 & 0.0 & 0.0 & 0.0 & 6.3 & 8.6 & 48.2 & 63.1 \\
\hline \multicolumn{11}{|l|}{ 2. Charges and interest $2 /$} \\
\hline PRGF interest & 0.0 & 0.2 & 0.3 & 0.3 & 0.3 & 0.3 & 0.3 & 0.3 & 0.2 & 2.0 \\
\hline Total obligations & 0.0 & 0.2 & 0.3 & 0.3 & 0.3 & 0.3 & 6.6 & 8.8 & 48.4 & 65.1 \\
\hline (percent of quota) & 0.0 & 0.1 & 0.2 & 0.2 & 0.2 & 0.2 & 4.0 & 5.4 & 29.9 & 40.2 \\
\hline \multicolumn{11}{|l|}{$\begin{array}{l}\text { Cumulative obligations } \\
\text { (existing and prospective) } \\
\text { 1. Principal }\end{array}$} \\
\hline GRA repurchases & 0.0 & 0.0 & 0.0 & 0.0 & 0.0 & 0.0 & 0.0 & 0.0 & 0.0 & 0.0 \\
\hline PRGF repayments & 0.0 & 0.0 & 0.0 & 0.0 & 2.5 & 8.3 & 18.0 & 20.2 & 72.5 & 121.5 \\
\hline \multicolumn{11}{|l|}{ 2. Charges and interest 2 / } \\
\hline PRGF interest & 0.3 & 0.5 & 0.5 & 0.5 & 0.5 & 0.5 & 0.5 & 0.4 & 0.2 & 4.1 \\
\hline SDR net charges & 0.2 & 0.7 & 0.7 & 0.7 & 0.7 & 0.7 & 0.7 & 0.7 & 5.3 & 10.2 \\
\hline Total obligations & 0.5 & 1.2 & 1.2 & 1.2 & 3.7 & 9.5 & 19.1 & 21.3 & 78.0 & 135.8 \\
\hline Outstanding Fund credit & 58.4 & 75.4 & 81.0 & 81.0 & 78.6 & 70.3 & 52.3 & 32.0 & $\ldots$ & $\ldots$ \\
\hline \multicolumn{11}{|l|}{$\begin{array}{l}\text { Outstanding Fund credit } \\
\text { In percent of: }\end{array}$} \\
\hline Exports of goods and services 4/ & 5 & 6 & 6 & 5 & 4 & 3 & 2 & 1 & $\ldots$ & $\ldots$ \\
\hline External public debt & 2 & 4 & 3 & 3 & 2 & 2 & 1 & 1 & $\ldots$ & $\ldots$ \\
\hline Gross official reserves & 1 & 1 & 2 & 2 & 2 & 2 & 1 & 1 & $\ldots$ & $\ldots$ \\
\hline GDP & 0 & 0 & 0 & 0 & 0 & 0 & 0 & 0 & $\ldots$ & $\ldots$ \\
\hline Quota & 36 & 47 & 50 & 50 & 49 & 43 & 32 & 20 & $\cdots$ & $\ldots$ \\
\hline \multicolumn{11}{|l|}{ Total Obligations } \\
\hline Exports of goods and services $4 /$ & 0.0 & 0.1 & 0.1 & 0.1 & 0.2 & 0.4 & 0.7 & 0.6 & $\ldots$ & $\ldots$ \\
\hline External public debt & 0.0 & 0.1 & 0.0 & 0.0 & 0.1 & 0.3 & 0.5 & 0.5 & $\ldots$ & $\ldots$ \\
\hline Gross official reserves & 0.0 & 0.0 & 0.0 & 0.0 & 0.1 & 0.2 & 0.4 & 0.5 & $\ldots$ & $\ldots$ \\
\hline GDP & 0.0 & 0.0 & 0.0 & 0.0 & 0.0 & 0.0 & 0.1 & 0.1 & $\ldots$ & $\ldots$ \\
\hline Quota & 0 & 1 & 1 & 1 & 2 & 6 & 12 & 13 & 48 & 84 \\
\hline
\end{tabular}

Source: Fund staff estimates and projections.

$1 /$ Years are calendar years, not fiscal.

2/ Projections are based on current interest rates for PRGF loans. The current SDR interest rate is assumed for net use of SDRs.

3/ Based on the proposed level and phasing of access, and subject to Executive Board approval.

4/ Excludes reexports. 


\section{ATTACHMENT I. ISLAMIC REPUbLiC OF AFGHANistan: LeTter OF INTENT}

Kabul, March 4, 2009

Mr. Dominique Strauss-Kahn

Managing Director

International Monetary Fund

Washington, D.C. 20431

Dear Mr. Strauss-Kahn,

The purpose of this letter and the attached Supplementary Memorandum of Economic and Financial Policies is to inform you about the implementation of our economic program supported by the Poverty Reduction and Growth Facility, set out policies for the period ahead, and request waivers of nonobservance of two performance criteria. We also request the sixth disbursement following the completion of the Fifth Review under the arrangement, an extension of the arrangement until March 2010, and a rephasing of disbursements, with the Sixth Review tied to end-June performance criteria and an additional Seventh Review tied to end-December performance criteria. The extension will allow us to implement pending reforms, boost revenue performance, demonstrate our commitment to economic stability, and reach the completion point under the Enhanced HIPC Initiative.

We met all but one of the September 2008 quantitative performance criteria. The floor on fiscal revenues was missed by a small amount. Owing to a technical delay, the structural performance criterion on the submission of audited financial statements of the state-owned electricity company DABM was not met on time. The submission will be a prior action for completion of the Fifth Review.

We experienced serious difficulties in revenue administration and expenditure overruns in recent months, which will make it impossible to meet two quantitative performance criteria corresponding to the Sixth Review due in March 2009: the floor on fiscal revenues and the ceiling on net central bank financing to the government. A new test date for the Sixth Review (June 2009) and ambitious quarterly revenue targets are proposed for the remainder of the extended arrangement.

The revenue problems were caused mainly by governance problems at customs and at the state-owned fuel company. We are implementing corrective measures, including personnel changes, and are pleased to report a turnaround in collection since January 2009. We intend to amend income tax legislation and produce by March 21, 2009 the first report on the 
implementation of a memorandum of understanding between the Ministry of Finance and the Ministry of Commerce and Industry. We have reached understandings with Fund staff that the last two measures will also be prior actions for completion of the Fifth Review. For 2009/10, we are committed to a strong recovery in revenues. In case of a revenue shortfall, we will reduce expenditures accordingly. We are also committed to reinvigorating the economic reform agenda and advancing the implementation of the Afghanistan National Development Strategy.

We believe that the policies and measures set in this memorandum are adequate to achieve the objectives of the program and we stand ready to take additional measures if needed. We will consult with the Fund on the adoption of further measures or any revision of policies in accordance with Fund procedures. In addition, we will provide the Fund the information required to assess progress in implementing the program. Lastly, we consent to the publication of the staff report for the Fifth Review under the PRGF arrangement, the Supplementary Memorandum of Economic and Financial Policies, and the Technical Memorandum of Understanding, including on the IMF's website.

Sincerely yours,

$/ \mathrm{s} /$

Omar Zakhilwal

Minister of Finance /s/

Abdul Qadeer Fitrat

Governor, Da Afghanistan Bank 


\section{ATtaChMENT II. SupPlementary Memorandum of ECONOMIC ANd FinanCIAL POLICIES FOR 2009/10}

March 4, 2009

1. The Government of Afghanistan's economic policies for 2009/10 are designed to consolidate the gains achieved under the PRGF arrangement; redress recent slippages; and set the basis for low inflation, strong growth, and fiscal sustainability. This memorandum describes the quantitative framework for the remainder of the extended program, including the proposed quantitative targets and structural conditionality for the Sixth and Seventh Reviews (Tables 1 and 2). The Sixth and Seventh Reviews will be completed on or after September 21, 2009 and March 19, 2010, respectively.

\section{A. Recent Developments and Outlook}

2. Growth and inflation. The economic and security situation has been challenging in 2008/09 owing to high food and fuel prices and increased violence in some provinces. A severe drought hit the agriculture sector, which accounts for about 30 percent of the economy, requiring emergency imports of wheat. Consequently, we estimate real GDP growth in 2008/09 to decline to about 3 percent and inflation to be about 27 percent on average.

3. Fiscal outturn. Due to weak revenues and higher than expected spending, we estimate that the operating budget deficit excluding grants will be Af 33 billion, instead of Af 20.5 billion as targeted. We also estimate that discretionary cash balances will amount to no less than Af 1.4 billion after cancellation of some wheat contracts compared to Af 4 billion in 2007/08. We were unable to observe the end-September 2008 quantitative performance criterion on domestic revenues (by a small margin), for which we are requesting a waiver. We expect not to observe the end-March 2009 quantitative performance criteria set at the time of the Fourth Review on domestic revenues and on net central bank financing of the government. We are setting new quarterly targets through March 2010, with performance criteria for June 2009 and December 2009 (Sixth and Seventh Reviews under the PRGFsupported program).

4. Revenues. We expect to collect total revenues in 2008/09 of Af 40 billion compared with Af 44.5 billion target. Performance has been good at the large and medium taxpayer offices in Kabul. We have extended these offices to Herat, Jalalabad, and Mazar-e-Sharif (endSeptember 2008 structural benchmark). In addition, traders have been notified that their exemption from the general income tax regime has expired (end-July 2008 structural benchmark). However, customs collection has been disappointing due to governance problems that led to poor enforcement and undervaluation of customs duties. Collection of duties on fuel 
entering through Hairatan (about 80 percent of total fuel imports) has been hampered by customs officers' lack of access to storage depots of the Fuel and Liquid Gas Enterprise (FLGE). In response to these problems, we have implemented personnel changes; set up audit units in Kabul, Kandahar, and Herat; strengthened the head office's control over the mobile verification teams; increased the head office's audit capacity; and introduced a daily reporting regime for identification of underperformance. We are already seeing a turnaround in collection, and we expect these and other measures (see below) to further improve collection in 2009/10. Lastly, legislation approving the introduction of the business receipts tax (BRT) on imports was delayed (end-November 2008 structural benchmark). We now expect legislative approval by midMarch 2009, which will enable collection of the BRT on imports later that month.

5. Expenditures. Spending pressures rose in the middle of 2008/09 due to worsening security, higher food and fuel prices, drought-related food shortages, and increases in teachers' salaries. Accordingly, the midyear budget review provided for: (i) Af 4.8 billion of wheat purchases; (ii) Af 1.3 billion for clearance of arrears by the Ministry of Education; (iii) Af 1 billion for salary increases for the military and police; and (iv) Af 2.2 billion in salary increases for teachers. The salary increase for teachers was financed by donors and accelerates the pay and grade reforms of the civil service.

6. Public enterprises. Reforming public enterprises continues to face serious challenges. Privatization suffered setbacks when Parliament stopped the auction of property of the Agricultural Development Bank in December. The attempt to privatize Afghan Telecom has also been unsuccessful. We will move forward to privatize FLGE and, despite delays, we will conclude and publish its financial audit, covering about 80 percent of operations by end-June 2009.

7. Electricity subsidies. We were unable to prevent Da Afghanistan Breshna Moasisa/Sherkat (DABM/S), the state electricity company, from spending the annual fuel subsidy about halfway through the 2008/09 fiscal year. Furthermore, the expenditure occurred without sufficient assurances as to its efficient use and accountability. The endSeptember 2008 structural performance criterion on the submission of DABM/S audited financial statements for 2006/07-2007/08 was not observed and will be a prior action for the completion of the Fifth Review. With some delay, we concluded a plan to reduce subsidies from the Ministry of Finance to DABM/S on February 22, 2009 (end-December 2008 structural benchmark).

8. Fiscal relations with public enterprises. We have prepared a preliminary review of fiscal relations between the government and key public enterprises, but due to data limitations and capacity constraints, the review could not include an action plan for three of the four enterprises (end-December 2008 structural benchmark). We note that although Ariana continues to delay tax payments and has problems with debts on fuel and airport fees, it has not benefited from loans by state-owned banks or government guarantees. 
9. Monetary policy. We have consistently observed the ceilings on currency in circulation. We have continued to foster the growth of the capital notes (central bank bills) market in order to strengthen control over monetary aggregates and encourage banks to use notes to manage liquidity. As first steps in launching the secondary market for notes, the central bank, Da Afghanistan Bank (DAB), has created an electronic registry of notes (endSeptember 2008 structural benchmark) and allowed banks to use them as collateral for shortterm interbank lending. DAB will install an upgraded electronic registry by May 2009. In June 2008 , to encourage banks to use notes to manage liquidity, we widened the interest corridor on standing facilities from $+/-150$ bps to $+/-350$ bps above/below the rate 28 -day notes.

10. Financial sector. Following the intervention in November 2008 of DAB in a small private bank, reserve requirements were tightened by excluding banks' cash in vault and current account deposits in DAB from eligible instruments and requiring banks to hold reserves in a "lock box" and to maintain required reserves daily. The central bank has also increased staffing at the financial supervision department, introduced an incentive-based salary scheme, separated onsite and offsite functions, and increased the frequency of onsite examinations to twice per year. We will soon introduce a credit bureau, a collateral registry, and possibly a limited deposit insurance scheme geared toward small depositors and funded by premiums from depository institutions. In August 2008, we prepared an action plan to address weaknesses identified in our self-assessment against Basel Principles (end-September 2008 structural benchmark) and are now implementing the plan.

11. DAB internal structural reforms. The external audit of DAB for end-2007/08 has been completed and posted on DAB's website. Among other things, the report provides assurance on the existence and availability of DAB's foreign reserves. As recommended by the safeguards assessment, we are actively recruiting an independent accounting expert to be appointed to the internal audit committee and a qualified deputy director and an advisor for the internal audit department. After consulting with commercial banks, we concluded that it was impractical to produce a monetary survey using a unified timeframe (end-June 2008 structural benchmark). We have instead produced a monetary survey based on a hybrid (Solar/Gregorian) timeframe, while ensuring concordance between key items reported simultaneously by the banks. Lastly, since October 2008, monetary data produced by DAB have been sourced directly from DAB's new accounting system. We are now working on ensuring consistency of the DAB's and Ministry of Finance's data on government deposits.

12. HIPC Completion Point. We intend to work toward reaching the HIPC completion point. To regularize relations with external creditors not covered under the Paris Club, we are pursuing agreements to settle our bilateral debts. We will honor our obligations under the 
Paris Club agreement and accelerate work toward meeting the completion point triggers including advancing the implementation of the Afghanistan National Development Strategy and preparing a debt sustainability analysis. We will further improve data on service exports and we expect to have survey-based service export estimates for the last two years by endJune 2009 for use in calculating debt relief.

13. Macroeconomic outlook. We project real GDP growth of 9 percent in 2009/10. Although the global downturn implies weaker export growth and a deceleration of industry and services activities, a recovery in agricultural output will be sufficient to sustain high growth. Lower commodity prices and a disinflationary monetary program will lock in the downward inflation trend and enable us to reduce annual inflation to 6 percent at the end of 2009/10. We expect a current account deficit of about 3.7 percent of GDP, or 51 percent of GDP, if grants are excluded.

\section{B. Fiscal Policies for 2009/10}

14. Fiscal balance. The budget for 2009/10 attempts to balance the need to increase spending, mainly in security and education, with limited resources. We are targeting total domestic revenues of Af 51 billion and total operational expenditures of Af 84 billion. To help ensure that we achieve the revenue target, we will provide adequate funding for the logistics and operations of the tax and customs departments. Consequently, the operating deficit excluding grants will be Af 33 billion. We are targeting a balanced budget including grants. We also have a contingency for an additional Af 10.4 billion related to security spending, to be financed by external assistance.

15. Expenditures. Compared to $2008 / 09$, we have not budgeted for any additional wheat imports. We will contain the growth of domestically financed nonsecurity spending, including through slowing the implementation of pay and grade reforms. Should revenues fall short of the targets, we will cut expenditures accordingly to meet our fiscal deficit targets. We will not incur arrears. Through expenditure restraint and close monitoring of expenditure commitments and payments (see 120 ), we will ensure that commitments for wage expenditure are paid within one month of their due date; and commitments for goods, services, and capital expenditures, within three months of their due date.

16. Customs collections. Beyond the changes described in paragraph 4, we began implementing the memorandum of understanding between the Ministry of Finance and the Ministry of Commerce and Industry, granting customs officials full access to and control of fuel import consignments in Hairatan for weighing and valuation before these consignments are taken into custody by FLGE officials or stored in FLGE tanks. Starting March 21, 2009, 
customs will begin issuing monthly reports verifying that the memorandum of understanding has been implemented and documenting its effect on revenues, including a detailed assessment of the volume and value of all categories of fuel imported and the amount of taxes and customs duties levied and collected (prior action for the Fifth Review). We will also ensure that customs officials have continuous access to private fuel depots in Hairatan.

17. Other customs reforms. We will implement other measures to bolster customs enforcement. By end-April 2009, we will implement the Automated System for Customs Data (ASYCUDA) transit and declaration processing module in Nangarhar, Herat, and Kabul airport, and will extend the module to Nimroz by January 2010. We will implement the transit module along the Zaranj-Nimroz axis in the same period. By end-May 2009, we will set up post-clearance audit units in Jalalabad and Mazar-e-Sharif, to reinforce customs enforcement. Finally, to ensure better customs control at the border we will adopt and implement by endJanuary 2010 the business model of border controls clarifying the role of each ministry at the border in line with international best practice and in consultation with other stakeholders (Ministry of Commerce and Industry, and Ministry of the Interior).

18. Revenue administration. Building on recent progress, we will introduce the collection of BRT on imports at the point of entry effective March 21, 2009 (prior action). We will extend the large and medium taxpayers offices to more provinces, withdraw remaining trader concessions on filing and paying taxes, collect at least Af 79 million on airlines' liabilities, and step up our public campaign to increase taxpayers' awareness, including about the collection of BRT on imports at the point of entry. Starting July 2009, we will prepare monthly reports documenting collection of the BRT based on dutiable imports. The first report will be a structural benchmark for the Sixth Review. We remain committed to simplifying the trade regime by reducing the tariff on soft drinks from 40 percent to 20 percent, which was originally a performance criterion for the Sixth Review. This measure will be enacted through a presidential decree to be signed in September 2009 and implemented from March 2010 onwards.

19. Tax and customs departments. In consultation with Fund staff, the Ministry of Finance will prepare by end-December 2009 an action plan to improve the efficiency and effectiveness of the tax and customs department, particularly by enhancing their responsibility for human and financial resources under the authority of the Minister of Finance and by increasing the efficiency of their organizational structure.

20. Expenditure management. We will ensure that no arrears are incurred. Toward this end, we will improve cash management and expenditure execution and enhance our capacity to identify and prevent arrears. We have already improved the accuracy of the budget preparation process to improve calculations of expenditure commitments. We will also 
improve cash planning and management through credible spending ceilings and improved accounting and recording. Given our cash-based recording and reporting, we will need to establish auxiliary records to track and manage noncash transactions from commitments to payment authorizations. Accordingly, we will: (i) track monthly commitments by the Ministry of Finance and line ministries and ensure that these are within the approved spending ceilings/cash releases; (ii) regularly compile a consolidated statement of outstanding commitments and unpaid bills of all ministries and spending units as an input into cash planning and management by using appropriate thresholds; (iii) produce quarterly statements of the stock and flow of arrears starting from fiscal year 1387 (2008/09); and (iv) once these arrears are audited by Ministry of Finance internal audit office, we will establish a plan for their clearance in cash and not through mutual offset/netting.

21. Public enterprises. We will finalize, by end-January 2010, a comprehensive review of the financial situation of Ariana, Afghan Telecom, DABM/S, and FLGE and their fiscal relations with the government in terms of tax owed, subsidies (if applicable), and other payables of receivables. The review will include regularization of tax payments and other cross debts and reduction of subsidies (if applicable); closure of unauthorized bank accounts; review of corporate governance procedures; business plans; and plans for divestiture, restructuring, or privatization for these enterprises. Separately, starting end-November 2009, $\mathrm{DABM} / \mathrm{S}$ will publish quarterly financial flows and other key variables for Kabul and other jurisdictions for the preceding year and with a forecast for the coming year. The Ministry of Finance, as sole owner of the two state-owned banks, will take actions to improve their CAMEL ${ }^{1}$ ratings to comply with the central bank financial supervision department's recommendations by December 2009. Effective immediately, and in compliance with Decision No. 3 of April 17, 2007 of the Economic Committee of the Council of Ministers, we will replace FLGE's in-kind collection of the 2 percent service fee on fuel imports with the intended pecuniary service charge.

\section{Monetary and Financial Policies}

22. Monetary program. The $2009 / 10$ monetary program will focus on reducing inflation to single digits by June 2009 and to 6 percent by end-March 2010. In addition, the program provides for a healthy level of international reserves and encourages the growth of the secondary market for capital notes. Currency in circulation is targeted to grow by no more than 16 percent and net international reserves are targeted to increase by about US\$200 million. We also expect the stock of capital notes to increase from Af 14 billion in February 2009 to about Af 19 billion in March 2010. Inflation trends will be monitored

\footnotetext{
${ }^{1}$ Capital adequacy, Asset quality, Management, Earnings, and Liquidity.
} 
closely. Should inflation remain high, we will promptly act to tighten monetary policy and adjust the program in consultation with the Fund. We will also maintain a managed floating exchange rate policy to benefit from the role of the exchange rate as a shock absorber and to ensure the effectiveness of our monetary targeting.

23. Financial sector policies. We will strengthen DAB's supervisory capacity and push weaker banks to improve their CAMEL ratings. We will build up bank examiners' capacity, especially for auditing IT systems in commercial banks. With assistance from the World Bank, we will initiate the automation of offsite examination and supplement the full-scope examination cycle for banks with CAMEL ratings of 4 or worse with 2-3 targeted examinations focusing on implementation of actions stipulated in warning letters. Finally, we will work on measures to improve the two operating state banks' CAMEL ratings, especially with regard to the quality of their management. 
Table 1. Islamic Republic of Afghanistan: Quantitative Performance Criteria and Indicative Targets Under the PRGF Arrangement, 2009/10 1/

(Cumulative changes from March 20, 2009; unless otherwise indicated)

\begin{tabular}{|c|c|c|c|c|}
\hline & $\begin{array}{c}\text { Jun. 21, } 2009 \\
\text { Performance } \\
\text { Criteria }\end{array}$ & $\begin{array}{c}\text { Sept. 22, } 2009 \\
\text { Indicative } \\
\text { Target }\end{array}$ & $\begin{array}{c}\text { Dec. 21, } 2009 \\
\text { Performance } \\
\text { Criteria }\end{array}$ & $\begin{array}{l}\text { Mar. 20, 2010 } \\
\text { Indicative } \\
\text { Target }\end{array}$ \\
\hline & \multicolumn{4}{|c|}{ (In billions of Afghanis) } \\
\hline Floor on fiscal revenues of the government & 12.3 & 25.1 & 37.3 & 51.0 \\
\hline Ceiling on currency in circulation & 2.9 & 7.1 & 9.6 & 12.0 \\
\hline Ceiling on net central bank financing of the government & 0.1 & 0.0 & 3.0 & 8.4 \\
\hline $\begin{array}{l}\text { Indicative target (ceiling) on the operating budget deficit } \\
\text { of the government, excluding grants }\end{array}$ & 4.5 & 12.6 & 21.3 & 32.8 \\
\hline \multirow[t]{2}{*}{ Indicative target (ceiling) on reserve money } & 3.2 & 7.8 & 11.3 & 14.8 \\
\hline & \multicolumn{4}{|c|}{ (In millions of US dollars) } \\
\hline Floor on net international reserves of DAB & 58.7 & 127.6 & 166.9 & 197.5 \\
\hline $\begin{array}{l}\text { Ceiling on contracting or guaranteeing new medium- and } \\
\text { long-term nonconcessional external debt by } \\
\text { the government and DAB } 2 /\end{array}$ & 0.0 & 0.0 & 0.0 & 0.0 \\
\hline $\begin{array}{l}\text { Ceiling on short-term external debt owed or guaranteed by the } \\
\text { government or DAB } 2 /\end{array}$ & 0.0 & 0.0 & 0.0 & 0.0 \\
\hline $\begin{array}{l}\text { New external payments arrears, excluding interest on } \\
\text { preexisting arrears } 2 /\end{array}$ & 0.0 & 0.0 & 0.0 & 0.0 \\
\hline \multicolumn{5}{|l|}{$\begin{array}{l}\text { Ceiling on lending from state-owned banks to public enterprises } \\
\text { in need of restructuring or government guaranteeing } \\
\text { borrowing by these public enterprises } 2 /\end{array}$} \\
\hline & \multicolumn{4}{|c|}{ (In billions of Afghanis) } \\
\hline \multicolumn{5}{|l|}{ Memorandum items: } \\
\hline Operating budget deficit of the government, including grants & -1.1 & -2.2 & -1.7 & -0.1 \\
\hline \multicolumn{5}{|l|}{ Reference projections for the adjustors } \\
\hline Core budget development spending & 6.6 & 19.8 & 33.0 & 52.8 \\
\hline $\begin{array}{l}\text { External financing of the core budget and sale } \\
\text { or transfers of nonfinancial assets }\end{array}$ & 10.9 & 32.4 & 51.4 & 77.3 \\
\hline $\begin{array}{l}\text { Externally financed expenditures transferred to the } \\
\text { core operating budget }\end{array}$ & 0.0 & 0.0 & 0.0 & 0.0 \\
\hline
\end{tabular}

Source: Fund staff estimates.

1/ The performance criteria and indicative targets envisaged under the program, and their adjustors, are defined in the attached Technical Memorandum of Understanding.

2/ These performance criteria apply on a continuous basis. 
Table 2. Islamic Republic of Afghanistan: Structural Conditionality Under the PRGF Arrangement March 2009-January 2010

Fifth Review

Target Dates

Prior Actions

Submission of DABM/S audited financial statements for 2006/07-07/08.

Amendment of income tax legislation to introduce business receipts tax on imports.

Customs will prepare first monthly report verifying that the memorandum of understanding between the Ministry of Finance and the Ministry of Commerce and Industry has been implemented and its effect on revenue, including a detailed assessment of the volume and value of all categories of fuel imported and amount of taxes and customs duties levied and collected. The report will also document the access received by customs and any areas that need improvement.

\section{Sixth Review}

\section{Benchmarks}

Implementation of the ASYCUDA transit and Declaration Processing Module in Nangarhar, Herat, and Kabul Airport inland clearing depots.

Setting up of post clearance audit units in the Customs Directorates of Jalalabad and Mazar-e-Sharif.

Publication of the external audit report of FLGE for the period 2002/03-2007/08.

June 30,2009

Issuance of first monthly report by the revenue department verifying the collection of BRT on imports.

April 30, 2009

May 31, 2009

June 30, 2009

\section{Seventh Review 1/}

\section{Benchmarks}

DABM/S will publish bimonthly report on financial flows and other key variables.

Finalize a comprehensive review of the financial situation of Afghan Telecom, Ariana, DABM, and FLGE and their fiscal relations with the government in terms of tax owed, subsidies (if applicable), and other payables or receivables. The review will include regularization of tax payments and other cross debts and reduction of subsidies (if applicable); closure of unauthorized bank accounts; review of corporate governance procedures; business plans; and plans for divestiture, restructuring, or privatization for these enterprises.

Adopt and implement the business model of border controls clarifying the role of each ministry at the border consistent with internationally accepted best practices in consultation with other stakeholders (Ministry of Commerce and Industry and Ministry of the Interior) for better customs controls at the border.

Implement the ASYCUDA transit module along Zaranj-Nimroz axis and Declaration January 31,2010 Processing Module at Nimroz.

January 31, 2010

1/ Structural benchmarks for the Seventh Review will be revisited at the time of the Sixth Review. 


\section{AtTACHMEnt III. TeChNiCAL Memorandum OF Understanding}

1. This memorandum updates the understanding between the Afghan authorities and Fund staff in relation to the monitoring of the PRGF-supported program until March 2010. It defines selected structural performance criteria and benchmarks (Section I), valuation for monitoring quantitative targets under the program (Section II), quantitative performance criteria and indicative targets (Section III); and adjustors (Section IV). It also specifies the agreed data reporting (Section V).

\section{Structural Performance Criteria and Benchmarks}

2. Prior actions for the Fifth Review, and structural performance criteria and benchmarks for the Sixth and Seventh Reviews are specified in Table 2 of the Supplementary Memorandum of Economic and Financial Policies (SMEFP). The following section elaborates only on those measures that require specification.

\section{A. Fifth Review}

Specification of selected prior actions for completion of the Fifth Review:

- $\quad$ Amend income tax legislation to introduce business receipts tax (BRT) on imports. This includes passage by the Upper House of Parliament (as it has already been approved by the Lower House), and presidential ratification of the law allowing for its implementation from March 21, 2009.

- Customs will prepare the first monthly report verifying that the memorandum of understanding (MOU) between the Ministry of Finance and the Ministry of Commerce and Industry has been implemented. The report will gauge its effect on revenue, including a detailed assessment of the volume and value of all categories of fuel imported and amount of taxes and customs duties levied and collected. The report will also document the access received by customs, and any areas that need improvement.

\section{B. Sixth Review}

Specification of structural benchmarks for the Sixth Review:

- $\quad$ Prepare first monthly report by the revenue department verifying the collection of BRT on imports. The BRT is expected to be collected starting at the beginning of 1388 (2009/10), and as such, the collection will be verified as part of the regular report on revenues on an ongoing basis.

- $\quad$ Ministry of Finance (Customs Department) will implement the Automated System for Customs Data (ASYCUDA) transit and Declaration Processing Module in Nangarhar, Herat, and Kabul Airport inland clearing depots by April 30, 2009. Impact of ASYCUDA 
implementation on collection of customs duties and other taxes collected on imports shall be regularly reviewed and analyzed through data generated from the ASYCUDA databases.

- $\quad$ Ministry of Finance (Customs Department) will set up Post Clearance Audit (PCA) units in the Customs Directorates of Jalalabad and Mazar-e-Sharif by May 31, 2009. This shall complete the first phase of implementation extending the scope of the post clearance audits to the five major customs directorates in the country. Ministry of Finance shall monitor the results of the audits to be conducted on a monthly basis. A report analyzing the full impact of the PCA units on increasing compliance with customs laws and improving customs clearance procedures shall be submitted to the Ministry of Finance by the Customs Department at the end of the year. The report shall also give details of the amount of unpaid government revenue detected, the amount recovered and the amount outstanding.

- $\quad$ Ministry of Finance (Customs Department) will establish continuous and effective customs controls at the private sector fuel storage facilities at Hairatan by June 30, 2009. This would be in addition to providing access to Customs staff at the FLGE owned premises as a result of the MOU signed between the Ministry of Finance and Ministry of Commerce and Industry in early-1387 (2008/09) and will strengthen government's efforts for closing potential loopholes in the collection of customs duty and other taxes on imported fuel. The controls shall be developed with the support of the TA provided to the customs department by donors in accordance with internationally accepted best practices suitably adapted to local conditions. Ministry of Finance will eventually authorize collection of customs duty at Hairatan to minimize risks of nonduty-paid fuel being diverted away from the Mazar-e-Sharif internal customs depot. The implementation status report of the agreed measure shall be submitted to the IMF by June 30, 2009.

\section{Seventh Review}

Specification of structural benchmarks:

- $\quad$ Da Afghanistan Breshna Moasisa/Sherkat (DABM/S) will start publishing quarterly financial flows and other key variables for Kabul and other jurisdictions, both for the preceding year and with a forecast for the coming year, with the first report published by November 30, 2009. The variables displayed will include sources of revenues and other receivables, power generation, expenditures, subsidies received, overall and primary balances, technical and nontechnical (theft) losses including own use, collection rates, revenues at 100 percent collection rates, and financing and other payables. Revenues and subsidies will distinguish among cash, credits, other government obligations, and payment of bills on behalf of DABM/S. The reporting will take place with a two month lag on a quarterly basis beginning with the period June-September 2009. The corresponding templates for reporting will be developed by the IMF with inputs from the authorities and international technical experts as needed. 
- With support from qualified external experts, finalize by January 31, 2010 a comprehensive review of the financial situation of Afghan Telecom, Ariana, DABM/S, and FLGE and their fiscal relations with the government in terms of tax owed, subsidies (if applicable), and other payables or receivables. The review will include regularization of tax payments and other cross debts and reduction of subsidies (if applicable). Specifically, the review would include (i) payment of bills and service charges by the line ministries; (ii) payment of taxes by these public enterprises; (iii) transfers of these public enterprises' profits to the single Treasury account; (iv) closure of unauthorized bank accounts; (v) review of corporate governance procedures detailing how decisions are made, approved, and reviewed and definition of the roles of external and internal auditors and directors, as well as the status of management information systems; (vi) business plans for these enterprises; and (vii) plans for divestiture, restructuring, or privatization.

- $\quad$ Ministry of Finance (Customs Department) will adopt and implement the business model of border controls clarifying the role of each ministry at the border in consultation with other stakeholders (Ministry of Commerce and Industry and Ministry of Interior) for better customs controls at the border. The basic framework for this business model will be developed in accordance with internationally accepted best practices with TA support already at the disposal of the Ministry of Finance. Ministry of Finance shall coordinate with the Ministry of Commerce and Industry, Ministry of Interior, Ministry of Transport and other ministries who have representation at the borders to arrive at an agreement. The Ministry of Finance will give a full implementation report focusing specifically on the changes impacting customs controls and physical placements, if any, by January 31, 2010.

- $\quad$ Ministry of Finance (Customs Department) will implement the ASYCUDA transit module along Zaranj-Nimroz axis and Declaration Processing Module at Nimroz by January 31, 2010. Ministry of Finance will provide the necessary resources to the ASYCUDA program to ensure its timely implementation.

\section{Program Exchange Rates and Gold Valuation}

3. Program exchange rates are used for formulating and monitoring quantitative targets. All foreign assets and liabilities denominated in U.S. dollars will be converted into Afghanis at a program exchange rate of 52.1375 Afghanis per U.S. dollar, which corresponds to the cash rate of December 19, 2008. Gold holdings will be valued at US\$838.28 per ounce, the price as of December 19, 2008. Assets and liabilities denominated in SDRs and in foreign currencies other than the U.S. dollar will be converted into U.S. dollars at their respective exchange rates of December 19, 2008, as reported in the following table. Gold holdings will be valued at US\$838.28 per ounce, the price as of December 19, 2008. 


Exchange Rate
U.S. dollar/Canadian dollar
U.S. dollar/U.A.E. dirham
U.S. dollar/Egyptian pound
U.S. dollar/Euro
U.S. dollar/Hong Kong dollar
U.S. dollar/Indian rupee
U.S. dollar/Pakistani rupee
U.S. dollar/Polish zloty
U.S. dollar/Iranian rial
U.S. dollar/Saudi rial
U.S. dollar/Russian ruble
U.S. dollar/Swiss franc
U.S. dollar/United Kingdom pound
U.S. dollar/SDR

Exchange Rate

dolla

U.S. dollat

U.S. dollar/Hong Kong dollar

U.S. dollar/Indian rupee

U.S. dollar/Pakistani rupee

U.S. dollar/Russian ruble

U.S. dollar/SDR

\section{Program Rate}

0.820200

0.272300

0.181800

1.391200

0.129020

0.021150

0.012598

0.336300

0.000101

0.266600

0.035490

0.906000

1.492000

1.545010

\section{Quantitative Performance Criteria ANd Indicative Targets}

4. The quantitative performance criteria for June and December 2009 specified in Table 1 of the SMEFP are:

- $\quad$ Floors on fiscal revenue of the central government and net international reserves (NIR); and

- $\quad$ Ceilings on currency in circulation $(\mathrm{CiC})$; net central bank financing (NCBF) of the central government; contracting and/or guaranteeing new medium- and long-term nonconcessional external debt by the government or the Da Afghanistan Bank (DAB), the central bank, (continuous); short-term external debt owed or guaranteed by the government or the DAB (continuous); accumulation of external payment arrears, excluding interest on preexisting arrears (continuous); lending from state-owned banks to, or government guaranteed borrowing by, public enterprises in need of restructuring (continuous).

The above variables constitute indicative targets for September 2009 and March 2010. In addition, the program includes the following indicative targets for the next four quarters:

- $\quad$ Ceilings on the operating budget deficit of the central government excluding grants and on reserve money.

\section{A. Currency in Circulation}

5. Currency in circulation is defined as total currency issued by the DAB. It excludes currency held in the presidential palace vault, in the DAB main vault, and in the vaults of all the provincial and district branches of the DAB. 


\section{B. Net Central Bank Financing of the Government}

6. Net central bank financing of the government is defined as the difference between the central bank's claims on the government and government deposits at the DAB. These deposits exclude deposits held at the DAB's branches because of the unavailability of reliable and timely data from the DAB's branches.

\section{Net International Reserves}

7. NIR are defined as reserve assets minus reserve liabilities of the DAB, both of which are expressed in U.S. dollars.

8. Reserve assets of the DAB, as defined in the fifth edition of the balance of payments manual (BPM5), are claims on nonresidents denominated in foreign convertible currencies, that are controlled by the $\mathrm{DAB}$, and are readily and unconditionally available for the $\mathrm{DAB}$ to meet balance of payments financing needs, intervention in exchange markets, and other purposes. They include DAB holdings of monetary gold, SDRs, Afghanistan's reserve position in the IMF, foreign currency cash (including foreign exchange banknotes in the vaults of the DAB, but exclude cash held in the DAB's branches), and deposits abroad (including balances on accounts maintained with overseas correspondent banks). Excluded from reserve assets are any assets that are pledged, collateralized, or otherwise encumbered; ${ }^{1}$ claims on residents; precious metals other than monetary gold; assets in nonconvertible currencies; illiquid assets; and claims on foreign exchange arising from derivatives in foreign currencies vis-à-vis domestic currency (such as futures, forwards, swaps, and options).

9. Reserve liabilities are defined as short-term (original maturity) foreign exchange liabilities of the $\mathrm{DAB}$ to nonresidents (held at $\mathrm{DAB}$ headquarters); all credit outstanding from the IMF; foreign currency reserves of commercial banks held at DAB headquarters; commitments to sell foreign exchange arising from derivatives (such as futures, forwards, swaps, and options); and all arrears on principal or interest payments to commercial banks, suppliers, or official export credit agencies.

\section{Revenues of the Central Government}

10. Revenues of the central government are defined in line with the Government Financial Statistics Manual (GFSM 2001) but on a cash accounting basis, excluding grants. Revenue is an increase in net worth of the central government (including its units in the provinces and agencies) resulting from a transaction. Revenues of the central government include taxes and other compulsory transfers imposed by central government units, property

\footnotetext{
${ }^{1}$ In particular, assets that are counterpart of the government's foreign currency deposits that back letters of credit are excluded from the reserve assets. However, assets that are counterpart of other government deposits are included in the reserve assets.
} 
income derived from the ownership of assets, sales of goods and services, social contributions, interest, fines, penalties and forfeits and voluntary transfers received from nongovernment other than grants. The definition for program monitoring excludes grants and other noncompulsory contributions received from foreign governments and international organizations; such transfers between central government units would be eliminated in the consolidation of the fiscal reports and not recorded as revenue. Receipts collected by central government on behalf of noncentral government units should not be counted as revenue (e.g., Red Crescent fees). Receipts from the sale of nonfinancial assets, such as privatization, and transactions in financial assets and liabilities, such as borrowing but excepting interest payments, are also excluded from the definition of revenue.

11. Revenues should be recognized on a cash basis and flows should be recorded when cash is received. The official Afghanistan Government Financial Management Information System (AFMIS) reports will be used as the basis for program monitoring. Exceptional advanced payments will be treated as if received on the normal due date. All revenue must be supported by the relevant documentation and revenue receivables, where a cash sum has been recorded but the revenue item has not yet been accounted for, and revenues payable, where the revenue has been reported but the cash has yet to be recorded should be separately reported on a gross basis.

\section{E. External Debt and Arrears}

12. As set forth in point No. 9 of the Guidelines on Performance Criteria with Respect to Foreign Debt (Decision No. 12274-00/85; August 24, 2000), the term "debt" will be understood to mean a current (i.e., not contingent) liability, created under a contractual arrangement through the provision of value in the form of assets (including currency) or services, and which requires the obligor to make one or more payments in the form of assets (including currency) or services, at some future point(s) in time; these payments will discharge the principal and/or interest liabilities incurred under the contract.

- $\quad$ Debts can take a number of forms, the primary ones being: (i) loans, (i.e., advances of money to obligor by the lender made on the basis of an undertaking that the obligor will repay the funds in the future-including deposits, bonds, debentures, commercial loans and buyers' credits - and temporary exchanges of assets that are equivalent to fully collateralized loans under which the obligor is required to repay the funds, and usually pay interest, by repurchasing the collateral from the buyer in the future (such as repurchase agreements and official swap arrangements); (ii) suppliers' credits (i.e., contracts where the supplier permits the obligor to defer payments until some time after the date on which the goods are delivered or services are provided); and (iii) leases (i.e., arrangements under which property is provided that the lessee has the right to use for one or more specified period(s) of time that are usually shorter than the total expected service life of the property, while the lessor retains the title to the property). Excluded from this limit are leases of real property by Afghan embassies or other foreign representations of the government. 
- $\quad$ For the purpose of the guideline, the debt is the present value (at the inception of the lease) of all lease payments expected to be made during the period of the agreement excluding those payments that cover the operation, repair, or maintenance of the property. Arrears, penalties, and judicially-awarded damages arising from the failure to make payment under a contractual obligation that constitutes debt are debt. Failure to make payment on an obligation that is not considered debt under this definition (e.g., payment on delivery) will not give rise to debt.

13. The ceiling on medium- and long-term external debt applies on a continuous basis to the contracting or guaranteeing by the government or the DAB of new nonconcessional external debt with an original maturity of more than one year. For program purposes, "government" includes the central government (including government departments), as well as official agencies that do not seek profit and whose budgets are issued independent of the annual operational or development budgets. Consistent with the Public Finance and Expenditure Management (PFEM) Law, the Ministry of Finance should have sole responsibility for the contracting and guaranteeing of external debt on behalf of the government.

- It applies to both debt as defined in paragraph 12 of this memorandum, and also to commitments contracted or guaranteed for which value has not been received. For the purposes of the program:

- $\quad$ external debt will be considered to have been contracted at the point the loan agreement or guarantee is signed by the Ministry of Finance (on behalf of the government) or the DAB Governor; and

- $\quad$ the guarantee of a debt arises from any explicit legal obligation of the government or the DAB, or any other agency acting on behalf of the government, to service such a debt in the event of nonpayment by the recipient (involving payments in cash or in kind), or indirectly through any other obligation of the government or the DAB to cover a shortfall incurred by the loan recipient.

- $\quad$ Excluded from the limits are refinancing credits and rescheduling operations, credits extended by the IMF, and credits on concessional terms defined as those with a grant element of at least 60 percent. The grant element is to be calculated using currency-specific discount rates based on the Organization for Economic Cooperation and Development's Commercial Interest Reference Rates (CIRRs): for maturities of less than 15 years, the grant element will be calculated based on six-month averages of CIRRs; and for maturities longer than 15 years, the grant element will be calculated based on 10 year averages.

- $\quad$ Debt falling within the limit shall be valued in U.S. dollars at the exchange rate prevailing at the time the contract or guarantee becomes effective.

14. The zero ceiling on short-term external debt applies on a continuous basis to the stock of short-term external debt owed or guaranteed by the government (as defined in 
paragraph 14 of this memorandum) or the $\mathrm{DAB}$, with an original maturity of up to and including one year.

- $\quad$ It applies to debt as defined in paragraph 12 of this memorandum.

- $\quad$ Excluded from the limit are rescheduling operations (including the deferral of interest on commercial debt) and normal import-related credits.

- $\quad$ Debt falling within the limit shall be valued in U.S. dollars at the exchange rate prevailing at the time the contract or guarantee becomes effective.

15. A continuous performance criterion applies to the nonaccumulation of new external payments arrears on external debt contracted or guaranteed by the central government or the DAB. External payment arrears consist of external debt service obligations (principal and interest) falling due after March 20, 2006 and that have not been paid at the time they are due, as specified in the contractual agreements. Excluded from the prohibition on the accumulation of new arrears are: (i) arrears arising from interest on the stock of arrears outstanding as of March 20, 2006; and (ii) external arrears that are subject to debt rescheduling agreements or negotiations.

\section{F. Lending to, or Guaranteeing Borrowing by, Public Enterprises}

16. The zero ceiling on new lending from state-owned banks to, or government guaranteed borrowing by public enterprises in need of restructuring applies on a continuous basis.

- For the purposes of this performance criterion:

- "state-owned banks" refers to those banks that are wholly or majority owned by the government (as defined in paragraph 14 of this memorandum); including Bank Millie, Bank Pashtany, and Export Promotion Bank;

- "enterprises in need of restructuring" refers to enterprises that meet any one of the following: (i) enterprises (public or private) that have not had an audited balance sheet in fiscal years 1384 and 1385; (ii) public enterprises that have been identified by the Ministry of Finance for liquidation; (c) public enterprises that do not have Cabinet-approved restructuring plans;

- " "public enterprises" refers to enterprises wholly or majority owned by the government, including those covered by the State-Owned Enterprise (Tassady) Law, 
and 13 state-owned corporations ${ }^{2}$ and any other public entities and government agencies engaged in commercial activities but not covered by Tassady Law.

- It applies to any new loans (or financial contributions) extended directly from stateowned banks to public enterprises in need of restructuring, and also to any new government guarantees (as defined in paragraph 13 of this memorandum) of borrowing undertaken by these public enterprises. It applies to loan agreements and guarantees for which value has not been received.

\section{G. Operating Budget Deficit of the Central Government, Excluding Grants}

17. Revenues of the central government as defined above minus operating budget expenditure recorded in AFMIS.

\section{H. Reserve Money}

18. Reserve money is defined to include $\mathrm{CiC}$ and Afghani-denominated commercial bank deposits with the DAB, including balances maintained by the commercial banks in the DAB's overnight facility.

\section{AdJUSTORS}

19. The floor on NIR and the ceiling on the NCBF of the government are consistent with the assumption that core budget development spending in 2009/10 will amount, on a cumulative basis from March 21, 2009, to:

$$
\begin{array}{ll}
\text { June 20, 2009 } & \text { Af } 6.6 \text { billion } \\
\text { September 21, 2009 } & \text { Af } 19.8 \text { billion } \\
\text { December 20, 2009 } & \text { Af 33.0 billion } \\
\text { March 20, 2010 } & \text { Af 52.8 billion }
\end{array}
$$

Should core budget development spending exceed these projections, the NIR floor will be adjusted downward and the NCBF ceiling will be adjusted upward by the difference between the actual level (up to the appropriated amount) and the projected level of development spending.

20. The NIR floor and NCBF ceiling are defined consistent with the assumption that the external financing of the core budget and receipts from the sale or transfer of nonfinancial assets will amount, on a cumulative basis from March 21, 2009, to:

\footnotetext{
${ }^{2}$ Afsotar, Afghan Teor, Aftento, Af-Turk, Afghan Cart, Afghan Naichi, Astrass, Afghan Telecom, Afghan Wireless, Afghan National Insurance Company, Afghan Textile, Ariana Afghan Airlines, and Hotel Intercontinental (Baghi Bala/Kabul).
} 
June 20, 2009

September 21, 2009

December 20, 2009

March 20, 2010
Af 10.9 billion

Af 32.4 billion

Af 51.4 billion

Af 77.3 billion

Should external financing of the core budget (including that associated with off-budgetary spending coming on budget) and the receipts from the sale or transfer of nonfinancial assets collectively exceed (fall short of) these projections, the NIR floor will be adjusted upward (downward) and the NCBF ceiling will be adjusted downward (upward) by the difference between their actual level and the projected level.

\section{Should some expenditure currently financed directly by donors outside the} budget be moved on to the operating budget, the NIR floor will be adjusted downward, and the NCBF ceiling and the indicative targets (ceilings) for the operating budget deficits of the central government, excluding grants, will be adjusted upward, by the actual amount of these expenditures on the conditions that (i) the moving on budget of these expenditures is justified by a statement from donors indicating their decision to stop financing them outside the budget and (ii) they are subject to a supplementary appropriation approved by parliament. The overall downward adjustment to the NIR floors will be capped at US\$300 million.

22. Should the central government undertake security-related operating expenditures based on spending plans agreed between the central government and donors, the indicative target (ceiling) for the operating budget deficit of the central government, excluding grants, will be adjusted upward and, should there be quarterly discrepancies between disbursements and executions, the NIR floor will be adjusted downward, and the NCBF ceiling upward by the sum of: (i) Af 1.8 billion, contingent on the final 2009/10 Afghan National Army (ANA) headcount exceeding 86,000, with the adjustment for any force size between 86,000 and 109,000 calculated on a prorated basis; (ii) Af 4.5 billion for augmentations ("top-ups") to the salaries of the 109,000 ANA force size with the adjustment for any force size between 86,000 and 109,000 calculated on a prorated basis; (iii) Af 2.2 billion for the salaries of the 16,000 soldiers recruited in 2008/09; (d) Af 900 million for equipment purchases by the Ministry of Defense; (iv) Af 400 million for equipment purchases by the Ministry of the Interior; and (v) Af 599 million for increasing the food allowances of the Afghan National Police. The cumulative downward adjustment to the NIR floors at each test date will be capped at US\$199.5 million. The cumulative upward adjustment to the NCBF ceilings and the indicative targets (ceilings) for the operating budget deficit of the central government, excluding grants, at each test date, will be capped at Af 10.4 billion.

\section{Provision of Information to the Fund}

23. To facilitate monitoring program implementation, the government of Afghanistan and the DAB will provide Division A of the Middle East and Central Asia Department through the office of the Resident Representative of the IMF in Afghanistan, the information 
specified below and summarized in the list of reporting tables provided by to the Technical Coordination Committee (see paragraph 25 below).

24. Actual outcomes will be provided with the frequencies and lags indicated below.

- DAB net international reserves: weekly, no later than two weeks after the end of the week.

- Monetary statistics, including exchange rates, government accounts with the DAB, currency in circulation, reserve money, and a monetary survey: monthly and no later than three weeks after the end of the month. The monetary survey will include the balance sheet of the DAB and a consolidated balance sheet of the commercial banking sector.

- Core budget operations and their financing: monthly and no later than four weeks after the end of the month. The official reports for the purpose of program monitoring will be the monthly financial statements from the Afghanistan Financial Management Information System. The structure of financing (grants and loans should be separately identified) and expenditure data should be on a consistent cash basis. Core operating expenditures should be reported on a monthly basis using the budget appropriation economic (object) and administrative classification in addition to the program and functional classification as reported in the budget documents. Core development expenditures should also be reported separately on a monthly basis using the budget program classification in addition to the economic (object), administrative and functional classification consistent with the operating budget. All the data should also compare outturns against the approved budget. Core operating and development revenues and expenditures should also be reported by province, separately on the same monthly basis.

- $\quad$ External budget operations and their financing (i.e., donor funded spending outside the core budget treasury systems): semi-annually (more frequently if possible) and no later than eight weeks after the end of the period. External development expenditures should be reported on a disbursement basis (as currently defined in budget documents) using the budget program classification (and an administrative, functional and provincial classification where possible).

- External debt data: quarterly and no later than six weeks after the end of the quarter. These will include: (i) details of new loans contracted or guaranteed during the quarter, including the terms of each new loan; (ii) the stock of debt at the end the quarter, including short-term debt, and medium- and long-term debt; (iii) loan disbursements and debt service payments (interest and amortization) during the quarter, beginning with the first quarter of fiscal year 1388 (2009/10); (iv) debt relief received during the quarter; (v) information on all overdue payments on short-term debt, and on medium- and long-term debt, including new external arrears (if any); and (vi) total outstanding amount of arrears. 
- National accounts data: annually and no later than eight weeks after the end of the year. Merchandise trade data should be reported quarterly and no later than eight weeks after the end of the quarter.

- Consumer price indexes (CPIs) for the city of Kabul and for Kabul and five other major cities ("national" CPI): monthly and no later than four weeks after the end of the month.

25. The Technical Coordination Committee (TCC) will prepare and send to the IMF reports by the end of each quarter explaining progress in implementing structural performance criteria and benchmarks under the program. These reports will include appropriate documentation to substantiate progress achieved, and explain any deviations relative to the initial reform plans or timetable, specifying expected revised completion date. Other details on major economic and social measures taken by the government that are expected to have an impact on program sequencing (such as changes in legislation, regulations, or any other pertinent document) will be sent in a timely manner to IMF staff, for consultation or information. 


\title{
INTERNATIONAL MONETARY FUND
}

ISLAMIC REPUBLIC OF AFGHANISTAN

\section{Fifth Review Under the Three-Year Arrangement Under the Poverty Reduction and Growth Facility and Request for Waiver of Performance Criteria, and Rephasing and Extension of the Arrangement}

\author{
Informational Annex \\ Prepared by Middle East and Central Asia Department \\ (In consultation with other Departments) \\ April 7, 2009 \\ Contents \\ Page

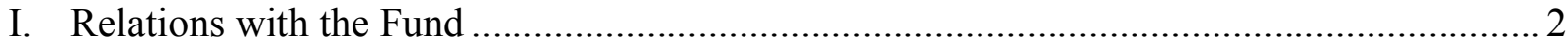

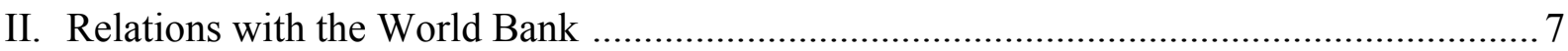 \\ III. Relations with the Asian Development Bank ............................................................... 9
}




\section{ANNeX I. ISLAMic RePUblic OF AfGHANistan-Relations With THE FUnd}

(As of February 28, 2009)

I. Membership Status: Joined July 14, 1955; Article XIV.

II. General Resources Account

Quota

Fund holdings of currency

Reserve position in Fund

Holdings Exchange Rate

III. SDR Department

Net cumulative allocation

Holdings

IV. Outstanding Purchases and Loans

PRGF Arrangements

\begin{tabular}{rr} 
SDR Million & \% Quota \\
\hline 161.90 & 100.00 \\
161.92 & 100.01 \\
0.00 & 0.00
\end{tabular}

\begin{tabular}{rr} 
SDR Million & \% Allocation \\
\hline 26.70 & 100.00 \\
0.03 & 0.11
\end{tabular}

$\underline{\text { SDR Million }}$

58.40 36.07

\section{Latest Financial Arrangements:}

\begin{tabular}{|c|c|c|c|c|}
\hline & $\begin{array}{l}\text { Date of } \\
\text { Arrangement }\end{array}$ & $\begin{array}{l}\text { Expiration } \\
\text { Date }\end{array}$ & $\begin{array}{l}\text { Amount Approved } \\
\text { (SDR Million) }\end{array}$ & $\begin{array}{l}\text { Amount Drawn } \\
\text { (SDR Million) }\end{array}$ \\
\hline & June 26, 2006 & June 25,2009 & 81.00 & 58.40 \\
\hline
\end{tabular}

\section{Projected Payments to Fund}

(SDR million; based on existing use of resources and present holdings of SDRs)

Principal

Charges/Interest

Total
Forthcoming

\begin{tabular}{lcc}
\multicolumn{3}{c}{ Forthcoming } \\
\hline$\underline{2009} \quad \underline{2010} \quad \underline{2011}$
\end{tabular}

$\begin{array}{lllll}\underline{0.41} & \underline{0.45} & \underline{0.45} & \underline{0.44} & \underline{0.41} \\ \underline{0.41} & \underline{0.45} & \underline{0.45} & \underline{2.89} & \underline{8.70}\end{array}$

\section{Implementation of HIPC Initiative:}

Enhanced

Framework

I. Commitment of HIPC assistance

Decision point date

July 2007

Assistance committed (NPV terms) ${ }^{1}$

by all creditors (US\$ million)

571.40

Of which: Fund assistance (US\$ million)

(SDR equivalent in million)

\footnotetext{
${ }^{1}$ Net Present Value (NPV) at the decision point under the enhanced framework.
} 
Completion point date

Floating

II. Disbursement of IMF assistance (SDR million)

Assistance disbursed to the member

Interim assistance

Completion point balance

Additional disbursement of interest income ${ }^{2}$

Total disbursements

VIII. Implementation of MDRI Assistance: Not Applicable

\section{Nonfinancial Relations}

\section{Exchange Arrangement}

Afghanistan is an Article XIV member country. The authorities are implementing a liberal exchange system. Based on information currently available to the staff, no exchange restrictions and multiple currency practices are in place. The authorities have provided documents to Fund staff related to laws and regulations on the exchange regime and have requested technical assistance from the Fund to formalize the current liberal regime. They have been implementing a managed float system with no predetermined path for the exchange rate. On March 24, 2009, the average of the buying and selling exchange rates in cash transactions on the Kabul money exchange market was Af 51.49 per US\$1.

To conduct monetary policy, the authorities rely on foreign exchange auctions since May 2002, and on short-term capital note auctions since September 2004. The foreign exchange auctions were initially open only to licensed money changers, but since June 2005 they are also open to commercial banks. The capital note auctions are open to commercial banks. Auctions are linked to the overall monetary program and are held on a regular basis (biweekly for foreign exchange auctions, daily for capital note auctions).

\section{Article IV Consultation}

The last Article IV consultation with Afghanistan was discussed by the Executive Board on February 13, 2008. Consultations with Afghanistan are on the standard 24-month cycle for the Fund's program countries.

\footnotetext{
${ }^{2}$ Under the enhanced framework, an additional disbursement is made at the completion point corresponding to interest income earned on the amount committed at the decision point, but not disbursed during the interim period.
} 


\section{Safeguards Assessment}

Under the Fund's safeguards assessment policy, Da Afghanistan Bank (DAB) is subject to a safeguards assessment with respect to PRGF arrangement approved on June 26, 2006. A safeguards assessment of DAB was completed on June 12, 2006 and updated on March 18, 2008. The assessment revealed serious vulnerabilities in DAB's external and internal audit mechanisms, as well as in its financial reporting framework and system of internal controls. Capacity constraints, however, hampered the effective implementation of measures recommended by the assessment, and follow-up staff visits found that while external audit arrangements had been strengthened, financial reporting and accounting controls remain weak. DAB is continuing its implementation of outstanding safeguards recommendations. These efforts are being monitored by staff in the context of the program.

\section{Technical Assistance, 2005-09}

\begin{tabular}{|c|c|c|}
\hline Department & Date & Purpose \\
\hline \multirow[t]{11}{*}{ FAD } & March 2004-July 2006 & Resident treasury expert \\
\hline & May $19-25,2005$ & Seminar on reorganization of the MOF \\
\hline & October $18-25,2005$ & Tax policy \\
\hline & February 21-March 4, 2006 & Public financial management \\
\hline & April 30-May 9, 2006 & Tax policy \\
\hline & November $15-23,2006$ & Tax and customs administration \\
\hline & August $18-30,2007$ & Program budgeting \\
\hline & August 28-September 12, 2007 & GFS \\
\hline & October 2007-September 2008 & Resident Treasury Advisor \\
\hline & June 24-July 8, 2008 & Program Budgeting \\
\hline & January 27-31, 2009 & Tax Administration \\
\hline \multirow[t]{3}{*}{ FIN } & April 2-11, 2006 & Safeguards assessment \\
\hline & November 9-12, 2007 & Safeguards assessment \\
\hline & September $20-24,2008$ & Safeguards assessment \\
\hline LEG & January 29-February 12,2005 & Income tax law \\
\hline \multirow[t]{2}{*}{ LEG/MCM } & May $2-10,2006$ & $\begin{array}{l}\text { Legal framework of DAB's monetary and payment system } \\
\text { policies }\end{array}$ \\
\hline & January $13-21,2008$ & Monetary policy and payment system \\
\hline \multirow[t]{4}{*}{ MCM } & September 2004-April, 2006 & Training coordinator \\
\hline & April 2006-June 2007 & Training coordinator (peripatetic) \\
\hline & February $2-15,2005$ & $\begin{array}{l}\text { Implementation of DAB balance sheet reconstruction and } \\
\text { capital adequacy recommendations }\end{array}$ \\
\hline & May $16-25,2005$ & Monetary policy and financial markets \\
\hline \multirow[t]{10}{*}{$\mathrm{MCM}$} & October $11-20,2005$ & Monetary policy \\
\hline & November $20-29,2005$ & Financial markets \\
\hline & January 22-February 5, 2006 & Monetary policy \\
\hline & January 22-February 5, 2006 & Monetary policy \\
\hline & May $2-10,2006$ & $\begin{array}{l}\text { Monetary policy, foreign reserve management, money } \\
\text { markets and insurance }\end{array}$ \\
\hline & November 28-December 10, 2006 & Monetary policy formulation and implementation \\
\hline & March 19-April 16, 2007 & Central bank capacity building \\
\hline & March 24-29, 2007 & Banking supervision \\
\hline & April 17-30, 2007 & Monetary policy formulation and implementation \\
\hline & November 7-14, 2007 & Banking Supervision \\
\hline
\end{tabular}




\begin{tabular}{|c|c|c|}
\hline Department & Date & Purpose \\
\hline \multirow[t]{23}{*}{ STA } & January 15-February 15,2005 & Multisector statistics \\
\hline & March 23-June 11, 2005 & Multisector statistics \\
\hline & July 20-August 31, 2005 & Multisector statistics \\
\hline & October 31, 2005-January 12, 2006 & Multisector statistics \\
\hline & March 6-15, 2006 & Balance of payments statistics \\
\hline & March 8-May 2, 2006 & Multisector statistics \\
\hline & March 13-April 1, 2006 & Monetary and financial statistics \\
\hline & May 24-July 18, 2006 & Multisector Statistics \\
\hline & August 2-16, 2006 & Balance of payments statistics \\
\hline & August 8-October 3, 2006 & Multisector statistics \\
\hline & September 22-October 4, 2006 & Government finance statistics \\
\hline & October 21-November 5, 2007 & Balance of payments statistics \\
\hline & November 1-December 26, 2006 & Multisector statistics \\
\hline & November 5-19, 2006 & Monetary and financial statistics \\
\hline & April 22-May 4, 2007 & Monetary and financial statistics \\
\hline & July 7-30, 2007 & National accounts \\
\hline & August 29-September 25, 2007 & National accounts \\
\hline & March 30-April 2, 2008 & TA evaluation \\
\hline & April 20-30, 2008 & Monetary and financial statistics \\
\hline & August 8-27, 2008 & Balance of payments statistics \\
\hline & October $13-29,2008$ & National accounts \\
\hline & November 12-December 3, 2008 & Balance of payments statistics \\
\hline & November 23-December 5, 2008 & Monetary and financial statistics \\
\hline MCD & April 16-20, 2005 & Basic macroeconomic accounting and financial programming \\
\hline \multirow[t]{3}{*}{ METAC } & November $15-20,2005$ & Tax policy and administration \\
\hline & June $8-13,2006$ & Budget integration \\
\hline & November $15-22,2006$ & Tax policy and administration \\
\hline \multirow[t]{21}{*}{ METAC } & December 12-19, 2006 & Budget integration \\
\hline & December 12-22, 2006 & Budget development \\
\hline & March 10-21, 2007 & Tax policy and administration \\
\hline & March 11-22, 2007 & Cash management \\
\hline & March 24-29, 2007 & Banking supervision \\
\hline & May $19-25,2007$ & Balance of payments statistics \\
\hline & June 6-19, 2007 & Accounting \\
\hline & July $1-12,2007$ & Banking supervision \\
\hline & August 2007 & Cash management and program budgeting \\
\hline & September $1-12,2007$ & Banking supervision \\
\hline & November $4-8,2007$ & Banking supervision \\
\hline & January 5-26, 2008 & Central bank accounting \\
\hline & March 22-April 2, 2008 & Banking supervision \\
\hline & June 25-July 7, 2008 & Budget preparation \\
\hline & August 6-27, 2008 & Balance of payments statistics \\
\hline & August 24-27, 2008 & Banking supervision \\
\hline & September 14-24, 2008 & Real sector statistics \\
\hline & December $14-17,2008$ & Banking supervision \\
\hline & January 25-February 2, 2009 & Central bank accounting \\
\hline & January 28-February 2, 2009 & Tax policy and administration \\
\hline & March 7-19, 2009 & Cash and budget management \\
\hline
\end{tabular}


Afghanistan is a participant in the Middle East Technical Assistance Center.

With financial support from the Sweden Technical Assistance Subaccount, the Fund sponsored a number of training activities aimed at DAB officials. A long-term resident

training advisor, Mr. Khan, was posted at DAB from September 2004-April 2006 and is now conducting peripatetic technical assistance missions. Finally, Sweden has financed a monetary policy peripatetic advisor (a position currently shared by Mr. Coats and Mr. Gray) to assist DAB in developing the strategy and instruments for improving the implementation of monetary policy.

\section{Resident Representatives}

Mr. de Schaetzen; August 2002-June 2005

Mr. Charap; June 2005-June 2008

Mr. Abdallah; June 2008-present 


\section{ANNEX II. ISLAMIC REPUBLIC OF AFGHANISTAN-RELATIONS WITH THE WORLD BANK}

(As of March 04, 2009)

1. Since 2002, the International Development Association (IDA) has committed a total of US\$1.7 billion in grants (74 percent) and credits (26 percent) in Afghanistan. Three budget support operations as well as 31 development and emergency reconstruction projects have been implemented to date. During FY2007-08, IDA delivered 10 new investment operations, three additional financing operations, and one US\$80 million budget support operation for a net commitment amount of US\$562 million. IDA's current active portfolio comprises 23 investment projects with combined net commitment of US\$991 million, of which US\$550 million (or 55 percent) had been disbursed as of January 31, 2009.

2. For this FY2009 (July 2008-June 2009), the Board is expected to approve eight new operations for a commitment amount of US\$240 million. Areas of intervention during this fiscal year include health service delivery, customs, rural livelihoods (the National Solidarity Program and Irrigation Rehabilitation) and natural resource management. A US\$30-35 million budget support operation is also envisaged to be delivered by the end of the fiscal year. In terms of sectoral composition of the overall investment portfolio, rural and transport sectors continue to represent the two largest shares, with respect to both commitments and disbursements. This is largely due to the successful implementation of the two nation-wide programs: National Solidarity Program (NSP) that supports development of community demand-driven rural infrastructure and community level governance, and the National Emergency Employment Program (NEEP) that provides short-term employment opportunities to the vulnerable segments of rural population through rural roads rehabilitation and reconstruction. A new two-year Interim Strategy Note is planned to be delivered in June 2009. It will support the recently released Afghanistan National Development Strategy.

3. The Afghanistan Reconstruction Trust Fund (ARTF) Management Committee (MC) consists of the World Bank (the administrator), the Islamic Development Bank (IDB), the Asian Development Bank (AsDB) and the UN (UNAMA and UNDP). The Government, represented by the Ministry of Finance, plays an active role in both the MC meetings (as an observer) and the donor meetings. During 2007-08, the ARTF handled US\$634 million of donor contributions. For 2008-09, donor pledges reached US\$695 million. The largest single contributors to the ARTF are the United Kingdom, the United States, and Canada. Other donors include 15 European countries, the European Commission, Australia, India, Iran, Turkey, Australia, and the Gulf States. The priority use of ARTF funds is the Government's operating budget (the Recurrent Cost Window) and secondarily for investment projects (the Investment Window). Since the ARTF's inception more than US\$1.6 billion has been disbursed to finance recurrent costs, and US\$741 million for investment projects. 
4. The World Bank has provided advice to help the government manage donor funds effectively and in a transparent way. The World Bank also supports the government by providing analytical work on the economy, public administration, gender, poverty, the opium economy, and an ongoing public finance management (PFM) review. The Bank has actively supported key reforms, particularly in the fiscal and public administration spheres, and through its budget support operation. It has advocated building capacity and legitimacy of the State and channeling donor resources through the government to ensure investments are aligned with national priorities. To this end, the World Bank works closely with other multilateral and bilateral agencies across a number of sectors where aid coordination and government ownership are most critical. 


\section{ANNEX III. ISLAMIC REPUbLiC OF AfGHANistan-RElations WITH THE ASIAN DEVELOPMENT BANK}

(As of March 12, 2009)

1. A new Country Partnership Strategy (CPS) covering the 2009-13 period was endorsed by the Asian Development Bank (AsDB)'s Board of Directors on March 5, 2009. The new CPS is fully aligned with the Afghanistan National Development Strategy (ANDS), with AsDB's investments contributing to Afghanistan's further economic development. The new CPS continues AsDB's narrow focus on Afghanistan's energy, transport, and agriculture and irrigation sectors, including sector governance and further institutional and human capacity development.

2. As of end-December 2008, the AsDB had committed an aggregate of some US\$1.77 billion to Afghanistan's reconstruction through approved Asian Development Fund (ADF) loan- and grant-financed projects, technical assistance activities, donor grant-financed projects administered by AsDB, and AsDB private sector investments. AsDB's ADFfinanced investments totaled US\$1.37 billion (US\$735 million in loans and \$630 million in grants). Since 2007, all of AsDB's public sector (ADF) assistance has been provided on a 100 percent grant basis. At the June 2008 International Conference in Support of Afghanistan AsDB pledged an additional $\$ 1.3$ billion over the 2008-13 period, making AsDB Afghanistan's fourth largest donor overall (after the United States, the United Kingdom, and the World Bank).

3. In November 2008 AsDB approved a US\$570 million energy sector multitranche financing facility (MFF) and a US\$400 million transport sector MFF; a US\$300 million water resources management MFF is planned for 2009. With the approval of these three MFFs, the bulk of AsDB's anticipated ADF assistance over the 2008-2013 will be fully "earmarked," although with some flexibility depending on changes in Government priorities.

4. Since AsDB operations in Afghanistan resumed in 2002, there have been 11 public sector loans and 13 grants covering 22 investment projects (13 loan or combined loan and grant projects, and 9 completely ADF grant-financed projects), all from ADF resources. In addition, there have been 8 separate and 2 combined donor-financed grant investment projects totaling US\$137.8 million. AsDB's private sector operations in Afghanistan began in 2004. As of end-2008, cumulative approvals for six private sector projects have amounted to US\$208.1 million.

5. The AsDB has been actively engaged in providing analytical and advisory technical assistance (TA) services to the Government, with total TA amounting to some US\$63 million as of end-2008 (including US\$5.6 million in donor cofinancing). Increasingly, TA is included as integral parts of AsDB's grant-based investment projects, with focus on public financial 
management, procurement, and anti-corruption as well as institutional and human capacity development. AsDB also has provided ongoing TA support to the Ministry of Finance.

6. AsDB is a member of the Afghanistan Compact/ANDS Joint Coordination Management Board (JCMB) and also is a member of the Afghanistan Reconstruction Trust Fund Management Committee. AsDB plays an active part in other donor coordination activities, including the JCMB Social and Economic Development Standing Committee, the Ministry of Finance's High Level Committee on Aid Effectiveness, the Inter-Ministerial Committee on Energy, and others. 


\section{Statement by the IMF Staff Representative on the Islamic Republic of Afghanistan}

\section{April 15, 2009}

1. This statement contains information on recent developments in the Islamic Republic of Afghanistan that has become available since the staff report was circulated to Executive Directors on April 7, 2009. The information does not alter the thrust of the staff appraisal.

2. Preliminary fiscal data for the fiscal year ending on March 20, 2009 show that domestic revenues totaled Af 41.6 billion (7 percent of GDP) compared to the projected figure of Af 40 billion (6.7 percent of GDP). The turnaround in collection reflects the recent administrative measures described in the Supplementary Memorandum of Economic and Financial Policies.

3. Headline inflation (year-on-year) fell to 3 percent in March 2009, down from 9 percent in February. Food inflation fell to 1 percent and nonfood inflation (a measure of core inflation) remained at 7 percent.

4. The structural benchmark on implementation of the ASYCUDA Transit and Declaration Processing Module in Nangarhar, Herat, and Kabul Airport, scheduled for April 30, 2009, was implemented at end-March. 
Press Release No. 09/137

FOR IMMEDIATE RELEASE

April 22, 2009
International Monetary Fund

Washington, D.C. 20431 USA

\section{IMF Executive Board Completes Fifth Review under the PRGF Arrangement with the Islamic Republic of Afghanistan}

The Executive Board of the International Monetary Fund (IMF) today completed the fifth review of the Islamic Republic of Afghanistan's performance under the economic program supported by the arrangement under a Poverty Reduction and Growth Facility (PRGF). The completion of the review allows for an immediate disbursement of SDR 11.3 million (about US\$16.7 million), which would bring total disbursements under the arrangement to SDR 69.7 million (about US\$103.2 million).

In completing the review, the Board also granted waivers for the nonobservance of (i) a September 2008 quantitative performance criterion related to the collection of fiscal revenues and; (ii) a September 2008 structural performance criterion related to the external audit of the state electricity company. The Board also approved a nine-month extension of the arrangement to end-March 2010 and a rephasing of disbursements.

The PRGF arrangement for the Islamic Republic of Afghanistan was approved in June 2006 for an amount equivalent to SDR 81.0 million (about US\$120 million) (see Press Release No. $\underline{06 / 144)}$.

Following the Executive Board's discussion, Mr. Murilo Portugal, Deputy Managing Director and Acting Chair, stated:

“Afghanistan's economic and security situation has been exceptionally challenging. A drought and worsening security cut economic growth to 3 percent in fiscal year 2008/09, while higher food prices drove average inflation up to more than 20 percent. Despite these adverse conditions, the authorities have pursued prudent monetary and financial sector policies which helped to lower inflation to single digit levels in recent months. A recovery in agricultural production is expected to boost economic growth to 9 percent in 2009/10. 
"Tax revenue collection in 2008 was adversely affected by the slowdown in economic growth and serious governance problems in customs as well as weak performance of regional tax offices. Since January 2009, the authorities have been intensifying reforms in order to boost revenue, and results are beginning to show. Continued efforts in this area will be required to put Afghanistan on a credible path toward fiscal sustainability and reduced dependence on aid.

“The authorities' economic program for 2009/10 seeks to lower inflation further, boost tax revenues, reduce corruption, and strengthen control over key public enterprises that have high costs and nontransparent operations. The authorities intend to maintain a flexible exchange rate.

"Substantial efforts will be needed to maintain economic stability and further modernize revenue administration, improve governance, and reform public enterprises. The IMF will continue to support the authorities' efforts to achieve these goals. Success will require, in addition to sound macroeconomic policies, an improvement in security conditions, progress in building the country's infrastructure, and reforms to stimulate private investment."

The PRGF is the IMF's concessional facility for low-income countries. PRGF loans carry an annual interest rate of 0.5 percent and are repayable over 10 years with a grace period of $5 \frac{1}{2}$ years on principal payments. 


\section{Statement by Jafar Mojarrad, Executive Director for the Islamic Republic of Afghanistan and Abdelali Jbili, Advisor to Executive Director April 22, 2009}

On behalf of our authorities, we thank staff for their hard work and dedication as well as the excellent report, and Management and the Board for their continued support. The authorities are also grateful to the international community for their support, as demonstrated in the Paris Conference in June 2008 and the more recent conference in The Hague. The Afghan authorities remain committed to implementing their PRGF-supported program and achieving the objectives of the Afghanistan National Development Strategy (ANDS).

While progress was achieved in a number of areas, as documented in the report, program implementation during the second half of 2008/09 has clearly demonstrated that more time is needed to tackle complex and challenging reforms to reach the completion point under the Enhanced HIPC Initiative. For these reasons, the authorities request an extension of the arrangement until March 2010 and the addition of a Seventh Review of the program.

\section{Performance under the PRGF}

Performance under the program must be seen against the background of an extremely challenging environment as well as the authorities' limited administrative capacity. Developments during the second half of 2008/09 were marked by several adverse factors: the drought reduced GDP growth to 3 percent compared to 12 percent in the previous year; the sharp rise in fuel and food import prices pushed CPI inflation to an estimated 27 percent on average; and emergency imports of wheat worsened the external current account balance. These factors have been compounded by a further deterioration in the security situation and governance problems at the customs and the state-owned fuel company, which the authorities are currently addressing.

Notwithstanding these adverse conditions, all prior actions and performance criteria (PC) have been met, except the end-September 2008 quantitative PC on domestic revenue, which was missed by a small margin and for which the authorities are seeking a waiver. A number of structural benchmarks have been met, while two others, requiring more time, have been folded into benchmarks for the Sixth and Seventh Reviews. As indicated in the Staff Statement, a structural benchmark scheduled for end April 2009, namely the implementation of the ASYCUDA Transit and Declaration Processing modules in three airports, has been met one month earlier.

Significant progress has been made in the monetary and financial sector areas. Currency in circulation has been kept below the program ceilings, reserve accumulation exceeded the program objective, and the Afghani has remained broadly stable. Moreover, Da Afghanistan 
Bank (DAB) has continued to promote the use of capital notes (CNs, or central bank bills) to facilitate the development of a secondary market for these notes and enhance control over monetary aggregates.

Program implementation in the fiscal area has been challenging especially because of problems at customs, but those are now being addressed. As a result, and as mentioned in the staff documents, there has been a substantial pick up in tax collection since December 2006. This reflects strong performance of the large taxpayers' offices in improving collection of taxes on goods and services, as well as the positive impact of the measures taken recently, including personnel changes in the customs department, the setting up of audit units, and the regular monitoring of revenue collection performance. On the expenditure side, emergency budgetary outlays in connection with the drought, additional military spending, and salary increases of teachers have pushed the operating budget deficit beyond program limits.

In our statement for the Board meeting on the fourth review of the program, we highlighted the difficulties and the additional time needed to complete relatively complex structural reforms. This has proven to be the case for the reform of public enterprises, including the privatization of the telephone company, the auctioning of property of the Agricultural Development Bank, and the reduction of electricity subsidies. The authorities intend to use the extension of the PRGF period to make steadfast progress in these crucial areas.

\section{Outlook and reform program for 2009/2010}

Despite Afghanistan's limited trade and financial links with the rest of the world, the economy will not be totally immune from the fallout of the global crisis. Spillover effects will be felt in 2009/10 in manufacturing and services, thereby reducing their contribution to growth, while the impact on exports and remittances could contribute to a worsening of the external current account balance. Nonetheless, due to the expected recovery in agriculture, staff project real GDP to grow by 9 percent. The proposed program for 2009/2010 is appropriately focused on making decisive progress in reducing the operating fiscal deficit, through a strong recovery of revenue and prudent spending policy. It also seeks to reduce inflation and to move forward in implementing the structural reforms envisaged in the ANDS.

\section{Fiscal policy}

The authorities are committed to addressing the revenue problems, and the budget for 2009/10 has targeted an increase in total domestic revenue to over 7 percent of GDP. The authorities have already started collecting the Business Receipt Tax (BRT) on imports at the point of entry, which is a prior action for this Fifth review. Additional measures are planned to enhance customs and tax collection and strengthen the revenue administration, as highlighted in paragraphs $17-19$ of the SMEFP, including steps to address governance and 
accountability issues through regular monitoring and reporting of revenue performance. In this regard, the sharp turnaround in revenue collection since January 2009 bodes well for the revenues in the new fiscal year.

Government spending will increase only moderately, taking into account the expected growth in domestically financed security expenditure and existing commitments for wages and salaries and capital spending. The authorities intend to monitor expenditure commitments and payments, and stand ready to cut spending to offset any revenue shortfalls and achieve the overall fiscal target.

\section{Monetary and financial sector policies}

The authorities will seek to maintain inflation at no more than 6 percent by March 2010. A prudent monetary policy, together with the recovery of agricultural output and the decline in food and fuel import prices, should help achieve this objective. Data for end- March 2009 shows that headline inflation already declined to 3 percent (year-on-year), as highlighted in the Staff Statement. The program provides for an adequate level of international reserves, and DAB will further use CNs as an instrument of liquidity management. The managed floating exchange rate system will be maintained, which will help absorb exogenous shocks, while DAB intervention will be limited to smoothing excessive volatility.

The authorities will continue to strengthen the legal and regulatory framework of the banking system, including plans to introduce a credit bureau, a collateral registry, and a limited deposit insurance scheme for small depositors. DAB will strengthen its supervisory capabilities to enhance its oversight over growing commercial bank activity. It also intends to take the necessary action to improve CAMEL ratings of two state-owned banks, as recommended by its financial supervision department.

Structural reforms

The Afghan authorities remain committed to advancing their structural reform agenda, as reiterated in the LOI and SMEFP. They have completed an audit of the state fuel company and intend to complete by end-January 2010 a comprehensive review of the financial situation of Ariana, Afghan Telecom, the electricity company (DABM/S), and Fuel and Liquid Gas Enterprise (FLGE). The review will help identify and address potential fiscal risks and will clarify the fiscal relations between these public enterprises and the government, thereby paving the way for regularization of cross debts and tax arrears and reduction of subsidies. The review will also cover governance procedures and plans for divestiture, restructuring, or privatization of these enterprises. Finally, the Ministry of Finance will focus its efforts on enhancing the effectiveness of the tax and customs departments, and improving cash management and expenditure execution. These efforts will be carried out in close consultation with Fund staff. 


\section{HIPC completion point}

The authorities are firmly committed to accelerate work toward meeting the completion point triggers under the Enhanced HIPC Initiative. They will honor their obligations under the Paris Club agreement and will pursue agreements with other creditors. The authorities' task in meeting the triggers will be facilitated by the proposed extension of the arrangement. 